\title{
Active macro-zone approach for incremental elastoplastic-contact analysis
}

\author{
F. Cucco, S. Terravecchia and L. Zito*,† \\ D.to di Ingegneria Civile, Ambientale, Aerospaziale dei Materiali, Università di Palermo, viale delle Scienze, \\ 90128 Palermo, Italy
}

\begin{abstract}
SUMMARY
The symmetric boundary element method, based on the Galerkin hypotheses, has found an application in the nonlinear analysis of plasticity and in contact-detachment problems, but both dealt with separately. In this paper, we want to treat these complex phenomena together as a linear complementarity problem.

A mixed variable multidomain approach is utilized in which the substructures are distinguished into macroelements, where elastic behavior is assumed, and bem-elements, where it is possible that plastic strains may occur. Elasticity equations are written for all the substructures, and regularity conditions in weighted (weak) form on the boundary sides and in the nodes (strong) between contiguous substructures have to be introduced, in order to attain the solving equation system governing the elastoplastic-contact/detachment problem. The elastoplasticity is solved by incremental analysis, called for active macro-zones, and uses the well-known concept of self-equilibrium stress field here shown in a discrete form through the introduction of the influence matrix (self-stress matrix). The solution of the frictionless contact/detachment problem was performed using a strategy based on the consistent formulation of the classical Signorini equations rewritten in discrete form by utilizing boundary nodal quantities as check elements in the zones of potential contact or detachment. Copyright (C) 2013 John Wiley \& Sons, Ltd.
\end{abstract}

Received 16 January 2013; Accepted 12 July 2013

KEY WORDS: multidomain SGBEM; elastoplasticity; contact/detachment; linear complementarity approach

\section{INTRODUCTION}

The boundary element method (BEM) has been utilized by several researches to deal with the elastoplastic contact problem in two-dimensional (2D) [1-6] and in three-dimensional (3D) space [7], working inside the collocation strategy through an iterative approach, but the non-symmetry of the operators employed suggests a formulation having the characteristics of symmetry.

This symmetric formulation, born in the last two decades of the previous millennium, was treated from the theoretical point of view, but only a few simple applications were shown, mainly because of the computational drawbacks due to the presence of strong singularities in the kernels of the double integrals characterizing the coefficients of the solving equation system. Recently, several of these drawbacks have been overcome, thus giving the possibility to perform all those research actions to make the BEM symmetric formulation more competitive.

In order to clarify the growth process of this formulation in the fields of mechanics, an Appendix has been introduced in this paper.

\footnotetext{
*Correspondence to: L. Zito, D.to di Ingegneria Civile, Ambientale, Aerospaziale dei Materiali, Università di Palermo, viale delle Scienze, 90128 Palermo, Italy.

${ }^{\dagger}$ E-mail: zitoliborio@gmail.com 
The present paper shows a strategy to perform the elastoplastic-contact/detachment analysis using the Symmetric Boundary Element Method in a simultaneous analysis obtained by solving the nonlinear problems of elastoplasticity and contact detachment by using the linear complementarity problem (LCP) in an incremental approach.

Elastoplastic problem. This research uses an approach for active macro-zones shown by the present authors as an incremental elastoplastic analysis problem in [8] and as a limit analysis in [9]. The approach for active macro-zones shows a more rational and innovatory strategy regarding both the theoretical and computational aspects of the symmetric Galerkin boundary element method (SGBEM).

In the plastic analysis carried out using the symmetric BEM, it is necessary to distinguish a computing phase for the elastic response to all the actions, including the volumetric ones (body forces and plastic strains), and a subsequent phase for plastic strain evaluation, stored during the loading process [8].

In a first phase the Somigliana Identities (SIs) of the displacements and the tractions, both evaluated on the boundary, are employed through a weighting process, based on the Galerkin hypotheses. In the subsequent phase the stresses have to be evaluated in each bem-element and a predictor-corrector process has to be performed in order to evaluate the plastic strains stored in the bem-elements where the stress violates the elastic yield domain.

In both phases, strong singular integrals are involved in the domain integrals when stresses and tractions caused by volumetric actions have to be evaluated [10-13].

Contact-detachment problem. On the basis of the boundary integral method, in its symmetric formulation, the frictionless unilateral contact between two elastic bodies was studied according to the Signorini formulation [14]. A boundary discretization by boundary elements of the two bodies in contact leads to an algebraic formulation in the form of a LCP, discussed by several authors [15-17].

Elastoplasticity and contact detachment. The analysis of two bodies in contact having elastoplastic behavior can be performed simultaneously, using an LCP analysis, alternating the contactdetachment phenomenon with the plasticity one. This proves to be advantageous when this analysis is carried out through the symmetric BEM, mainly for two reasons:

the contact-detachment process proves to have immediate execution because it is carried out through comparison between generalized quantities evaluated along the boundary elements and the reference values in weighted form;

at every step characterizing the previous phase, an elastoplastic analysis is made in accordance with the predictor-corrector strategy.

\section{ELASTOPLASTIC-CONTACT/DETACHMENT ANALYSIS BY BIEM (CONTINUUM APPROACH)}

The solution of the elastoplastic-contact/detachment problem can be obtained by considering the two subproblems separately, that is, elastoplasticity and contact/detachment. Both these problems are nonlinear: the plasticity physically and the contact/detachment geometrically. The nonlinear nature of these problems demands the use of an iterative strategy to define the correction conditions (contact or detachment) at every point on the boundary and the value of the plastic strain increment where the yield conditions have been disregarded. A direct approach can be used to analyze the contact/detachment problem [17] and to study the plastic phenomenon as a limit analysis [9].

The equations governing the elastoplastic-contact/detachment problem inside a continuum approach are the following:

- the integral equations satisfying the boundary and domain conditions,

- the Signorini equations regarding the contact/detachment conditions rewritten as LCP, and

- the constitutive relations for rate-independent plasticity. 


\subsection{Boundary integral equations}

Let a body having domain $\Omega$ and boundary $\Gamma$ be subjected to domain and boundary actions, the latter without any difference between known and unknown quantities. We deal with the analysis of this body by considering both the physical nonlinearity (plasticity) and the geometrical (contact/detachment), through a step-by-step analysis.

For this purpose, the SIs have to be introduced:

$$
\begin{gathered}
\mathbf{u}=\int_{\Gamma} \mathbf{G}_{u u} \mathbf{f} \mathrm{d} \Gamma+\int_{\Gamma} \mathbf{G}_{u t}(-\mathbf{u}) \mathrm{d} \Gamma+\int_{\Omega} \mathbf{G}_{u u} \overline{\mathbf{b}} \mathrm{d} \Omega+\int_{\Omega} \mathbf{G}_{u \sigma} \boldsymbol{\varepsilon}^{p} \mathrm{~d} \Omega \\
\mathbf{t}=\int_{\Gamma} \mathbf{G}_{t u} \mathbf{f} \mathrm{d} \Gamma+\int_{\Gamma} \mathbf{G}_{t t}(-\mathbf{u}) \mathrm{d} \Gamma+\int_{\Omega} \mathbf{G}_{t u} \overline{\mathbf{b}} \mathrm{d} \Omega+\int_{\Omega} \mathbf{G}_{t \sigma} \boldsymbol{\varepsilon}^{p} \mathrm{~d} \Omega \\
\boldsymbol{\sigma}=\int_{\Gamma} \mathbf{G}_{\sigma u} \mathbf{f} \mathrm{d} \Gamma+\int_{\Gamma} \mathbf{G}_{\sigma t}(-\mathbf{u}) \mathrm{d} \Gamma+\int_{\Omega} \mathbf{G}_{\sigma u} \bar{b} \mathrm{~d} \Omega+\int_{\Omega} \mathbf{G}_{\sigma \sigma} \boldsymbol{\varepsilon}^{p} \mathrm{~d} \Omega
\end{gathered}
$$

These provide the displacements, tractions and stresses in the unbounded domain caused by layered mechanical jumps $\mathbf{f}$ and double-layered kinematical ones $(-\mathbf{u})$, both acting along the boundary $\Gamma$, as well as by body forces $\overline{\mathbf{b}}$ and volumetric inelastic actions $\boldsymbol{\varepsilon}^{p}$ in $\Omega$. The operators $\mathbf{G}_{p q}$ are the Fundamental Solution matrices, whose symbology was introduced by Polizzotto in $[18,19]$ : the sub-indices $p=u, t, \sigma$ and $q=u, t, \sigma$ indicate the effect and the dual quantity in an energetic sense associated with the cause, respectively.

The body is subjected to actions in its plane: forces $\overline{\mathbf{f}}_{2}$ at the portion $\Gamma_{2}$ of free boundary, displacements $\overline{\mathbf{u}}_{1}$ imposed at the portion $\Gamma_{1}$ of constrained boundary, body forces $\overline{\mathbf{b}}$ and plastic strains $\varepsilon^{p}$ in $\Omega$.

In the hypothesis that the physical and geometrical characteristics of the body are zone-wise variables, an appropriate subdivision of the domain into substructures has to be introduced. This subdivision involves the introduction of interface boundaries $\Gamma_{0}$ between contiguous substructures, distinguished into macroelements having merely elastic behavior and bem-elements having elastoplastic behavior.

We want to obtain the elastic response to the external actions in terms of displacements $\mathbf{u}_{2}$ on $\Gamma_{2}$ and reactive forces $\mathbf{f}_{1}$ on $\Gamma_{1}$, but also in terms of displacements $\mathbf{u}_{0}$ and tractions $\mathbf{t}_{0}$ on the interface boundary $\Gamma_{0}$ and of the stresses $\sigma$ in the domain of each bem-element by using the mixed variable multidomain SGBEM approach [20,21]. Equations (1a) and (1b) are utilized in order to obtain, through the employment of weighted quantities according to the Galerkin strategy, the algebraic operator coefficients necessary to perform the analysis phase; Equation (1c) is utilized to provide the stress state at the Gauss points in the post-processing phase.

Equation (1) provide the problem solution inside the boundary integral equation method (BIEM) through the following Dirichlet and Neumann conditions:

$$
\begin{aligned}
\mathbf{u}_{1}=\overline{\mathbf{u}}_{1} & \text { on } \Gamma_{1} \\
\mathbf{t}_{2}=\overline{\mathbf{f}}_{2} & \text { on } \Gamma_{2}
\end{aligned}
$$

and through the regularity conditions between substructures

$$
\begin{aligned}
\mathbf{t}_{0}^{A} & =-\mathbf{t}_{0}^{B} \quad \text { on } \Gamma_{0} \\
\mathbf{u}_{0}^{A} & =\mathbf{u}_{0}^{B} \quad \text { on } \Gamma_{0}
\end{aligned}
$$

where $A$ and $B$ are two contiguous substructures, and where by $\Gamma_{1}, \Gamma_{2}$ and $\Gamma_{0}$ denote the constrained, free and interface boundaries, respectively. 


\subsection{Contact/detachment conditions}

Let the homogeneous elastic 2D solid bodies $A$ and $B$ be subjected to imposed displacements $\overline{\mathbf{u}}_{1}$ on $\Gamma_{1}$, to boundary forces $\overline{\mathbf{f}}_{2}$ on $\Gamma_{2}$, to body forces $\overline{\mathbf{b}}$ in $\Omega$ and to plastic strains $\boldsymbol{\varepsilon}^{p}$ in $\Omega$. We suppose that friction does not occur between the two bodies and that only simple contact or detachment is possible.

Let us introduce the coefficient $c$ characterizing the cohesion between the boundaries in contact $\Gamma_{0}^{A}$ and $\Gamma_{0}^{B}$, in the zone of potential detachment, and the distance vector $\boldsymbol{h}$ representing the distance between the corresponding points (reference gap) on the boundary nodes of $\Gamma_{2}^{A}$ and $\Gamma_{2}^{B}$, in the zone of potential contact.

Let us denote

- by $\mathbf{t}_{0}^{A}=-\mathbf{t}_{0}^{B}$ the stress vector acting between the contact points on the boundaries $\Gamma_{0}^{A}$ and $\Gamma_{0}^{B}$, respectively, $\mathbf{t}_{2}^{A}=\mathbf{t}_{2}^{B}=\mathbf{0}$ being verified on the free boundaries $\Gamma_{2}^{A}$ and $\Gamma_{2}^{B}$, and

- by $\mathbf{u}_{2}^{A} \neq \mathbf{u}_{2}^{B}$ the displacement vectors at the detached boundaries $\Gamma_{2}^{A}$ and $\Gamma_{2}^{B}, \mathbf{u}_{0}^{A}=\mathbf{u}_{0}^{B}$ being equal quantities between the displacements of the contact points on the common boundaries $\Gamma_{0}^{A}$ and $\Gamma_{0}^{B}$.

The boundary conditions of the contact-detachment problem are the following:

$$
\begin{gathered}
\mathbf{n}_{2}^{A}\left(\left(\mathbf{u}_{2}^{A}-\mathbf{u}_{2}^{B}\right)-\mathbf{h}\right) \leqslant 0, \quad c=0 \text { gap condition } \\
\mathbf{n}_{0}^{A} \mathbf{t}_{0}^{A}-c \leqslant 0, \quad \mathbf{h}=\mathbf{0} \text { contact condition } \\
{\left[\mathbf{n}_{2}^{A}\left(\left(\mathbf{u}_{2}^{A}-\mathbf{u}_{2}^{B}\right)-\mathbf{h}\right)\right]\left[\mathbf{n}_{2}^{A} \mathbf{t}_{2}^{A}\right]=0 \text { complementarity condition on } \Gamma_{2}} \\
{\left[\mathbf{n}_{0}^{A}\left(\mathbf{u}_{0}^{A}-\mathbf{u}_{0}^{B}\right)\right]\left[\mathbf{n}_{0}^{A} \mathbf{t}_{0}^{A}-c\right]=0 \text { complementarity condition on } \Gamma_{0}}
\end{gathered}
$$

valid at every point on the boundary, where $\mathbf{n}_{2}^{A}$ and $\mathbf{n}_{0}^{A}$ are the transposes of the normal vectors associated with the boundary $\Gamma_{2}^{A}$ and $\Gamma_{0}^{A}$ of the body A, respectively.

The detachment process is the following:

in the boundary zone marked by $\Gamma_{0}$, where the contact between the two bodies occurs $(c>0, \mathbf{h}=\mathbf{0})$, the following conditions must be verified: $\mathbf{n}_{0}^{A}\left(\mathbf{u}_{0}^{A}-\mathbf{u}_{0}^{B}\right)=0$ and $\mathbf{n}_{0}^{A} \mathbf{t}_{0}^{A} \leqslant c$. The detachment phenomenon is checked through the value assumed by the traction $\mathbf{t}_{0}^{A}$. Indeed, it occurs when the external action change produces a traction $\mathbf{t}_{0}^{A}$ whose value satisfies the condition $\mathbf{n}_{0}^{A} \mathbf{t}_{0}^{A} \geqslant c, c$ being the limit value. Therefore the latter condition must be considered as the beginning of the detachment process. In this case, the point in contact is divided into two points belonging to the bodies $A$ and $B$, and, as a consequence, it causes the rise of new free boundaries $\Gamma_{2}^{A}$ and $\Gamma_{2}^{B}$.

The contact process is the following:

Vice versa, in the boundary zone marked by $\Gamma_{2}$, where there is no contact between the two bodies $(c=0, \mathbf{h}>\mathbf{0})$, the following conditions have to be verified: $\mathbf{n}_{2}^{A}\left(\mathbf{u}_{2}^{A}-\mathbf{u}_{2}^{B}\right) \leqslant \mathbf{n}_{2}^{A} \mathbf{h}$ and $\mathbf{n}_{2}^{A} \mathbf{t}_{2}^{A}=0$. The contact phenomenon is checked through the values assumed by the displacements $\mathbf{u}_{2}^{A}$ and $\mathbf{u}_{2}^{B}$ of the boundaries $\Gamma_{2}^{A}$ and $\Gamma_{2}^{B}$ : indeed, it occurs when the external action change causes values of the displacements $\mathbf{u}_{2}^{A}$ and $\mathbf{u}_{2}^{B}$ to satisfy the following condition: $\mathbf{n}^{A}\left(\mathbf{u}_{2}^{A}-\mathbf{u}_{2}^{B}\right) \geqslant \mathbf{n}^{A} \mathbf{h}$. Therefore the latter condition must be considered as the beginning of the contact process. In this case the points on $\Gamma_{2}^{A}$ and $\Gamma_{2}^{B}$ become connected at a single point, and, as a consequence, this causes the rise of a new contact zone $\Gamma_{0}$.

\subsection{Constitutive relations for rate-independent plasticity and return mapping algorithm}

The governing equations of classical plasticity will now be briefly summarized.

The stress vector is related to the elastic strain $\left(\varepsilon-\varepsilon^{p}\right)$ through the standard elasticity tensor $\mathbf{D}$, that is,

$$
\boldsymbol{\sigma}=\mathbf{D}\left(\varepsilon-\varepsilon^{p}\right)
$$


The essential feature that characterizes the plastic constitutive law is that the stress must belong to the space of the admissible stresses:

$$
F[\sigma] \leqslant 0
$$

$F$ being the yield function.

The irreversible part of the plastic process is given by the evolution of the plastic strain $\dot{\boldsymbol{\varepsilon}}^{p}$. The evolution laws, called flow rule, can be defined by the following incremental law:

$$
\dot{\varepsilon}^{p}=\partial_{\sigma} F[\sigma] \dot{\lambda}
$$

where $\dot{\lambda}$ is the increment of the plastic multiplier and is defined by means of the following loading/unloading conditions:

$$
\dot{\lambda} \geqslant 0, \quad F[\sigma] \leqslant 0, \quad F[\sigma] \dot{\lambda}=0
$$

Let us consider the classical theory developed by Simo and Hughes [22], based on the elastoplastic operator split, where the implicit-backward Euler scheme is applied to Equation (7). The following equations, governing the elastoplastic analysis, can be obtained for the elastic predictor:

$$
\sigma_{n+1}^{*}=\sigma_{n}+\Delta \sigma_{n+1}
$$

and for the plastic corrector:

$$
\left\{\begin{array}{l}
\boldsymbol{\sigma}_{n+1}=\boldsymbol{\sigma}_{n+1}^{*}-\mathbf{D} \partial_{\boldsymbol{\sigma}_{n+1}} F\left[\boldsymbol{\sigma}_{n+1}\right] \Delta \lambda_{n+1} \\
F\left[\boldsymbol{\sigma}_{n+1}\right]=0
\end{array}\right.
$$

$\Delta \lambda$ being the plastic multiplier increment.

It is important to note that the same result can be obtained through the extremal path theory $[23,24]$ or by the closest point return mapping algorithm [22].

The indices $n$ and $n+1$ represent the previous and current load steps, and $\sigma^{*}$ the elastic (trial) stress. In the hypothesis of a plane strain state, Equation (10) represents a nonlinear equation system in the unknowns $\sigma_{n+1}, \Delta \lambda_{n+1}$.

In particular, with regard to the case of a $2 \mathrm{D}$ continuous solid and in the hypothesis of a plane strain condition, initial strain approach, associated plastic flow and von Mises yield law, Equation (10) can be rewritten in the following form:

$$
\left\{\begin{array}{l}
\boldsymbol{\sigma}_{n+1}-\boldsymbol{\sigma}_{n+1}^{*}+\mathbf{D} \boldsymbol{M} \boldsymbol{\sigma}_{n+1} \Delta \lambda_{n+1}=\mathbf{0} \\
\frac{1}{2} \boldsymbol{\sigma}_{n+1}^{T} \boldsymbol{M} \boldsymbol{\sigma}_{n+1}-\sigma_{y}^{2}=0
\end{array}\right.
$$

$\sigma_{y}$ being the yield stress and $\boldsymbol{M}$ a matrix of constants [24].

Equations (1)-(4) and (11) characterize the nonlinear elastoplastic-contact/detachment problem inside the continuum type approach.

\section{THE DISCRETE APPROACH OF THE ELASTOPLASTIC-CONTACT/DETACHMENT ANALYSIS BY MULTIDOMAIN SYMMETRIC GALERKIN BEM}

In this section, the same problems, described in the continuum field, have to be rewritten in the following inside a discrete approach using the multidomain SGBEM.

In order to perform the nonlinear analysis of an elastoplastic-contact/detachment problem, the discrete equations obtained will be modified in a substantial way through a strong variable condensation process, giving rise to some matricial relations, which connect the nodal displacements $\mathbf{U}_{2}$ on $\Gamma_{2}$, the nodal forces $\mathbf{F}_{0}$ on $\Gamma_{0}$ and the stresses $\sigma$ at the Gauss points, characterizing the contact, the detachment and plasticity phenomena, respectively, all as functions of the plastic strains $\varepsilon^{p}$ and of the load multiplier $\beta$, that is,

$$
\mathbf{U}_{2}=\mathbf{U}_{2}\left[\varepsilon^{p}, \beta\right] \quad \text { contact }
$$




$$
\begin{array}{lr}
\mathbf{F}_{0}=\mathbf{F}_{0}\left[\varepsilon^{p}, \beta\right] & \text { detachment } \\
\boldsymbol{\sigma}=\boldsymbol{\sigma}\left[\boldsymbol{\varepsilon}^{p}, \beta\right] & \text { elastoplasticity }
\end{array}
$$

The methodology will utilize some incremental procedures such as the LCP. In detail: Equation (12a) will be utilized for contact problem, Equation (12b) for the detachment one and lastly Equation (12c) in an approach for active macro-zones will characterize the elastoplastic problems.

\subsection{Multidomain symmetric Galerkin BEM}

This section shows a detailed description of the procedure utilized to obtain, through the SGBEM applied to multidomain problems, three elasticity equations governing the elastoplasticcontact/detachment problem.

The bodies into Figure 1 are discretized into macroelements and bem-elements. The generic substructure is characterized by the boundary $\Gamma$ distinguished into three parts, free $\Gamma_{2}$, constrained $\Gamma_{1}$ and interface $\Gamma_{0}$, but generally not all present.

If we introduce the SI of the displacements, tractions and stresses (Equations (1a) and (1b)) inside the Dirichlet and Neumann conditions (Equation (2a) and (2b)), the following boundary integral equations can be obtained:

$$
\begin{aligned}
& \mathbf{u}_{1}\left[\mathbf{f}_{1},-\mathbf{u}_{2}, \mathbf{f}_{0},-\mathbf{u}_{0}\right]+\mathbf{u}_{1}\left[\varepsilon^{p}\right]+\mathbf{u}_{1}\left[\overline{\mathbf{f}}_{2},-\overline{\mathbf{u}}_{1}^{P V}, \overline{\mathbf{b}}\right]+\frac{1}{2} \overline{\mathbf{u}}_{1}=\overline{\mathbf{u}}_{1} \\
& \mathbf{t}_{2}\left[\mathbf{f}_{1},-\mathbf{u}_{2}, \mathbf{f}_{0},-\mathbf{u}_{0}\right]+\mathbf{t}_{2}\left[\varepsilon^{p}\right]+\mathbf{t}_{2}\left[\overline{\mathbf{f}}_{2}^{P V},-\overline{\mathbf{u}}_{1}, \overline{\mathbf{b}}\right]+\frac{1}{2} \overline{\mathbf{f}}_{2}=\overline{\mathbf{f}}_{2}
\end{aligned}
$$

where a symbolic form has been used and where the typologies of the boundary are characterized by the pedeses introduced in the displacement and force vectors.

It is necessary to define the unknowns $\mathbf{u}_{0}$ and $\mathbf{t}_{0}$, related to the interface boundary $\Gamma_{0}$ through the following SI:

$$
\begin{aligned}
& \mathbf{u}_{0}=\mathbf{u}_{0}\left[\mathbf{f}_{1},-\mathbf{u}_{2}, \mathbf{f}_{0},-\mathbf{u}_{0}^{P V}\right]+\frac{1}{2} \mathbf{u}_{0}+\mathbf{u}_{0}\left[\varepsilon^{p}\right]+\mathbf{u}_{0}\left[\overline{\mathbf{f}}_{2},-\overline{\mathbf{u}}_{1}, \overline{\mathbf{b}}\right] \\
& \mathbf{t}_{0}=\mathbf{t}_{0}\left[\mathbf{f}_{1},-\mathbf{u}_{2}, \mathbf{f}_{0}^{P V},-\mathbf{u}_{0}\right]+\frac{1}{2} \mathbf{t}_{0}+\mathbf{t}_{0}\left[\boldsymbol{\varepsilon}^{p}\right]+\mathbf{t}_{0}\left[\overline{\mathbf{f}}_{2},-\overline{\mathbf{u}}_{1}, \overline{\mathbf{b}}\right]
\end{aligned}
$$

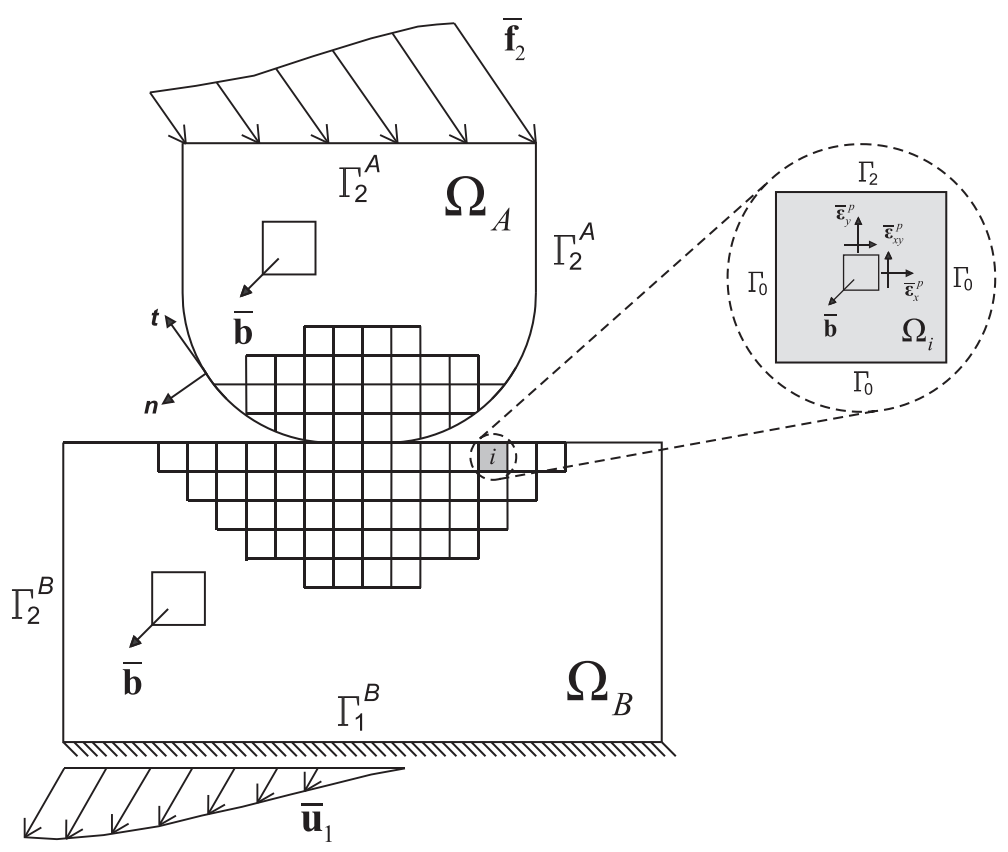

Figure 1. The body subdivided into substructures. 
and to introduce the stress field in the subdomain $\Omega$ of the bem-element being examined:

$$
\sigma=\sigma\left[\mathbf{f}_{1},-\mathbf{u}_{2}, \mathbf{f}_{0},-\mathbf{u}_{0}\right]+\sigma\left[\varepsilon^{p}\right]+\sigma\left[\overline{\mathbf{f}}_{2},-\overline{\mathbf{u}}_{1}, \overline{\mathbf{b}}\right]
$$

The terms $\mathbf{u}_{0}\left[-\mathbf{u}^{P V}\right]$ and $\mathbf{t}_{0}\left[\mathbf{t}^{P V}\right]$ define the integrals as Cauchy principal values (CPVs), and the terms where $1 / 2$ occurs are the corresponding free terms.

It is to be noted that Equations (13a) and (13b), and (14a) and (14b) were computed on a smooth boundary.

Equation (13a) and (13b) have to be rewritten in a different way:

$$
\begin{aligned}
& \mathbf{u}_{1}\left[\mathbf{f}_{1},-\mathbf{u}_{2}, \mathbf{f}_{0},-\mathbf{u}_{0}\right]+\mathbf{u}_{1}\left[\varepsilon^{p}\right]+\mathbf{u}_{1}\left[\overline{\mathbf{f}}_{2},-\overline{\mathbf{u}}_{1}^{P V}, \overline{\mathbf{b}}\right]-\frac{1}{2} \overline{\mathbf{u}}_{1}=\mathbf{0} \\
& \mathbf{t}_{2}\left[\mathbf{f}_{1},-\mathbf{u}_{2}, \mathbf{f}_{0},-\mathbf{u}_{0}\right]+\mathbf{t}_{2}\left[\boldsymbol{\varepsilon}^{p}\right]+\mathbf{t}_{2}\left[\overline{\mathbf{f}}_{2}^{P V},-\overline{\mathbf{u}}_{1}, \overline{\mathbf{b}}\right]-\frac{1}{2} \overline{\mathbf{f}}_{2}=\mathbf{0}
\end{aligned}
$$

whereas Equations (14a) and (14b), and (15) remain unchanged.

In the previous integral Equations (14), (15) and (16), $\boldsymbol{\varepsilon}^{p}$ is the plastic strain vector, whose evaluation strategy is defined in the next section.

We introduce the boundary discretization into the boundary elements by performing the following modeling of all the known and unknown quantities:

$$
\mathbf{f}_{1}=\Psi_{f} \mathbf{F}_{1}, \overline{\mathbf{f}}_{2}=\Psi_{f} \overline{\mathbf{F}}_{2}, \mathbf{f}_{0}=\Psi_{f} \mathbf{F}_{0}, \mathbf{u}_{2}=\Psi_{u} \mathbf{U}_{2}, \overline{\mathbf{u}}_{1}=\Psi_{u} \overline{\mathbf{U}}_{1}, \mathbf{u}_{0}=\Psi_{u} \mathbf{U}_{0}, \varepsilon^{p}=\Psi_{p} p
$$

where $\boldsymbol{\Psi}_{f}$ and $\boldsymbol{\Psi}_{u}$ are appropriate matrices of shape functions regarding the boundary quantities, whereas $\boldsymbol{\Psi}_{p}$ are domain shape functions used to model the volumetric plastic strains. Further, the capital letters indicate the nodal vectors of the forces $\left(\mathbf{F}_{1}, \overline{\mathbf{F}}_{2}\right.$ and $\left.\mathbf{F}_{0}\right)$ and of the displacements $\left(\overline{\mathbf{U}}_{1}, \mathbf{U}_{2}\right.$ and $\left.\mathbf{U}_{0}\right)$ defined on the boundary nodes.

We now perform the weighting of all the coefficients of Equations (14) and (16). For this purpose, the same shape functions as those modeling the causes are employed, but introduced in an energetically dual way in accordance to the Galerkin approach $[18,19,25,26]$, thus obtaining the following generalized equations:

$$
\int_{\Gamma_{1}} \psi_{f}^{T}\left(\mathbf{u}_{1}-\overline{\mathbf{u}}_{1}\right)=\mathbf{0}, \int_{\Gamma_{2}} \psi_{u}^{T}\left(\mathbf{t}_{2}-\overline{\mathbf{f}}_{2}\right)=\mathbf{0}, \quad \mathbf{W}_{0}=\int_{\Gamma_{0}} \psi_{f}^{T} \mathbf{u}_{0}, \mathbf{P}_{0}=\int_{\Gamma_{0}} \psi_{u}^{T} \mathbf{t}_{0}
$$

where Equation (18a) and (18b) symbolize the weighted forms of the Dirichlet and Neumann conditions, whereas Equation (18c) and (18d) are the weighted response in terms of displacements and tractions on the interface boundaries.

Equations (18a)-(18d) and (15) are rewritten in the following symbolic form:

$$
\left.\begin{array}{rl}
\mathbf{0} & =\mathbf{W}_{1}\left[\mathbf{F}_{1},-\mathbf{U}_{2}, \mathbf{F}_{0},-\mathbf{U}_{0}\right]+\mathbf{W}_{1}[\mathbf{p}]+\beta \hat{\mathbf{W}}_{1} \\
\mathbf{0} & =\mathbf{P}_{2}\left[\mathbf{F}_{1},-\mathbf{U}_{2}, \mathbf{F}_{0},-\mathbf{U}_{0}\right]+\mathbf{P}_{2}[\mathbf{p}]+\beta \hat{\mathbf{P}}_{2} \\
\mathbf{W}_{0} & =\mathbf{W}_{0}\left[\mathbf{F}_{1},-\mathbf{U}_{2}, \mathbf{F}_{0},-\mathbf{U}_{0}\right]+\mathbf{W}_{0}[\mathbf{p}]+\beta \hat{\mathbf{W}}_{0} \\
\mathbf{P}_{0} & =\mathbf{P}_{0}\left[\mathbf{F}_{1},-\mathbf{U}_{2}, \mathbf{F}_{0},-\mathbf{U}_{0}\right]+\mathbf{P}_{0}[\mathbf{p}]+\beta \hat{\mathbf{P}}_{0}
\end{array}\right\} \text { Symmetric BEM }
$$

where the load multiplier $\beta$ of the external actions has been introduced

In this way, it is possible to obtain the following block system regarding Equation (19a)-(19d):

$$
\begin{gathered}
\mathbf{0} \\
\mathbf{0} \\
\hline \mathbf{W}_{0} \\
\mathbf{P}_{0}
\end{gathered}|=| \begin{array}{cc|cc}
\mathbf{A}_{u 1 u 1} & \mathbf{A}_{u 1 f 2} & \mathbf{A}_{u 1 u 0} & \mathbf{A}_{u 1 f 0} \\
\mathbf{A}_{f 2 u 1} & \mathbf{A}_{f 2 f 2} & \mathbf{A}_{f 2 u 0} & \mathbf{A}_{f 2 f 0} \\
\mathbf{A}_{u 0 u 1} & \mathbf{A}_{u 0 f 2} & \mathbf{A}_{u 0 u 0} & \overline{\mathbf{A}}_{u 0 f 0} \\
\mathbf{A}_{f 0 u 1} & \mathbf{A}_{f o f 2} & \overline{\mathbf{A}}_{f 0 u 0} & \mathbf{A}_{f o f 0}
\end{array}|| \begin{gathered}
\mathbf{F}_{1} \\
-\mathbf{U}_{2} \\
\mathbf{F}_{0} \\
-\mathbf{U}_{0}
\end{gathered}|+| \begin{gathered}
\mathbf{A}_{u 1 \sigma} \\
\mathbf{A}_{f 2 \sigma} \\
\mathbf{A}_{u 0 \sigma} \\
\mathbf{A}_{f 0 \sigma}
\end{gathered}|\mathbf{p}|+\beta\left|\begin{array}{l}
\hat{\mathbf{W}}_{1} \\
\hat{\mathbf{P}}_{2} \\
\hat{\mathbf{W}}_{0} \\
\hat{\mathbf{P}}_{0}
\end{array}\right|
$$

In the latter block equation the matrix is symmetric. Moreover, the submatrices $\mathbf{A}_{i j}$ and the subvectors $\mathbf{W}, \mathbf{P}$ are formed by coefficients obtained through a double integration according to the 
SGBEM strategy. In detail, the first and second rows of the equation system represent the Dirichlet and Neumann conditions written in weighted form $\mathbf{W}_{1}-\overline{\mathbf{W}}_{1}=\mathbf{0}$ and $\mathbf{P}_{2}-\overline{\mathbf{P}}_{2}=\mathbf{0}$. The remaining rows regard the weighting of the displacements and tractions at the interface zones. The term $\overline{\mathbf{A}}_{u 0 f 0}=\overline{\mathbf{A}}_{f 0 u 0}^{T}$ includes the weighting of the CPV integrals and of the corresponding free terms.

The stress field is given by the following SI:

$$
\begin{aligned}
\boldsymbol{\sigma}= & \int_{\Gamma_{1}} \mathbf{G}_{\sigma u} \boldsymbol{\Psi}_{f} \mathrm{~d} \Gamma \cdot \mathbf{F}_{1}+\int_{\Gamma_{2}} \mathbf{G}_{\sigma t} \boldsymbol{\Psi}_{u} \mathrm{~d} \Gamma \cdot\left(-\mathbf{U}_{2}\right)+\int_{\Gamma_{0}} \mathbf{G}_{\sigma u} \boldsymbol{\Psi}_{f} \mathrm{~d} \Gamma \cdot \mathbf{F}_{0}+\int_{\Gamma_{0}} \mathbf{G}_{\sigma t} \boldsymbol{\Psi}_{u} \mathrm{~d} \Gamma \cdot\left(-\mathbf{U}_{0}\right)+ \\
& +\int_{\Omega} \mathbf{G}_{\sigma \sigma} \boldsymbol{\Psi}_{p} \mathrm{~d} \Omega \cdot \mathbf{p}+\beta\left(\int_{\Omega} \mathbf{G}_{\sigma u} \overline{\mathbf{b}} \mathrm{d} \Omega+\int_{\Gamma_{2}} \mathbf{G}_{\sigma u} \boldsymbol{\Psi}_{f} \mathrm{~d} \Gamma \cdot \overline{\mathbf{F}}_{2}+\int_{\Gamma_{1}} \mathbf{G}_{\sigma t} \boldsymbol{\Psi}_{u} \mathrm{~d} \Gamma \cdot\left(-\overline{\mathbf{U}}_{1}\right)\right)
\end{aligned}
$$

which translates in extensive form the Equation (19e). This latter, written in matricial form, takes one the form:

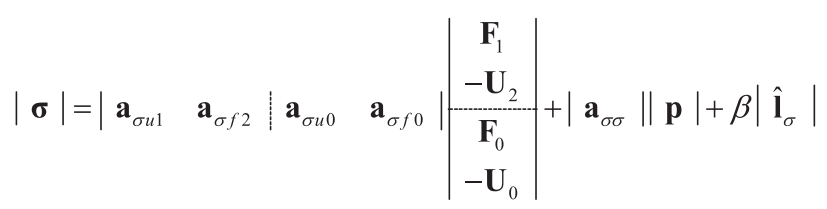

where the small letters are matrices containing coefficients obtained in accordance with collocation BEM.

In order to analyze the plastic phenomenon, the domain is discretized by introducing several bem-elements in the zones where the plastic strains have to be stored during the load-unload process.

In Equation (20), some coefficients show singular or hyper-singular kernels. These difficulties have been overcome within the SGBEM approach by using different techniques. We can mention the strategies of Bonnet [27], Frangi and Novati [28], Panzeca et al. [26], Terravecchia [29] and Holzer [30].

In Equation (21a), the singularities regard the coefficients of the matrix $\mathbf{a}_{\sigma \sigma}$ located on the diagonal, the latter providing the stress at the Gauss points due to plastic strain distribution $\varepsilon^{p}=\Psi_{p} \mathbf{p}$ in the same bem-elements. These integrals can be considered as CPVs with which the Bui free term [31] is associated. Otherwise, it is possible to employ the regularization technique with the aim of cutting off the strong singularity, followed by the radial integral method $[10,11]$ in order to permit the transformation of the domain integrals into boundary ones. This strategy has to be used in each bem-elements. The reader can refer to Panzeca et al. [12] and Zito et al. [13] for a more detailed discussion of the computational aspects and the related implementation techniques.

Equations (20) and (21b) can be expressed in compact form as follows:

$$
\begin{aligned}
\mathbf{0} & =\mathbf{A} \mathbf{X}+\mathbf{A}_{0} \mathbf{X}_{0}+\mathbf{A}_{\sigma} \mathbf{p}+\beta \hat{\mathbf{L}} \\
\mathbf{Z}_{0} & =\mathbf{A}_{0}^{T} \mathbf{X}+\mathbf{A}_{00} \mathbf{X}_{0}+\mathbf{A}_{0 \sigma} \mathbf{p}+\beta \hat{\mathbf{L}}_{0} \\
\boldsymbol{\sigma} & =\mathbf{a}_{\sigma} \mathbf{X}+\mathbf{a}_{\sigma 0} \mathbf{X}_{0}+\mathbf{a}_{\sigma \sigma} \mathbf{p}+\beta \hat{\mathbf{l}}_{\sigma}
\end{aligned}
$$

where

$$
\mathbf{Z}_{0}=\left|\begin{array}{c}
\mathbf{W}_{0} \\
\mathbf{P}_{0}
\end{array}\right|, \mathbf{X}=\left|\begin{array}{c}
\mathbf{F}_{1} \\
-\mathbf{U}_{2}
\end{array}\right|, \mathbf{X}_{0}=\left|\begin{array}{c}
\mathbf{F}_{0} \\
-\mathbf{U}_{0}
\end{array}\right|, \hat{\mathbf{L}}=\left|\begin{array}{c}
\hat{\mathbf{W}}_{1} \\
\hat{\mathbf{P}}_{2}
\end{array}\right|, \hat{\mathbf{L}}_{0}=\left|\begin{array}{c}
\hat{\mathbf{W}}_{0} \\
\hat{\mathbf{P}}_{0}
\end{array}\right|
$$

The vector $\mathbf{Z}_{0}$ collects the generalized (or weighted) displacement $\mathbf{W}_{0}$ and traction $\mathbf{P}_{0}$ subvectors defined at the interface boundaries, obtained as the response to all the known and unknown actions, regarding the boundary and domain quantities. The vector $\sigma$ collects the stresses, computed at the Gauss points, due to the same known and unknown actions.

By performing variable condensation through the replacement of the vector $\mathbf{X}$ extracted from Equation (22a) into Equation (22b) and (22c), one obtains

$$
\begin{aligned}
\mathbf{Z}_{0} & =\mathbf{D}_{00} \mathbf{X}_{0}+\mathbf{D}_{0 \sigma} \mathbf{p}+\beta \hat{\mathbf{Z}}_{0} \\
\boldsymbol{\sigma} & =\mathbf{d}_{\sigma 0} \mathbf{X}_{0}+\mathbf{d}_{\sigma \sigma} \mathbf{p}+\beta \hat{\boldsymbol{\sigma}}
\end{aligned}
$$


where one sets

$$
\begin{aligned}
& \mathbf{D}_{00}=\mathbf{A}_{0}^{T} \mathbf{A}^{-1} \mathbf{A}_{0}-\mathbf{A}_{00}, \mathbf{D}_{0 \sigma}=-\mathbf{A}_{0}^{T} \mathbf{A}^{-1} \mathbf{A}_{\sigma}+\mathbf{A}_{0 \sigma}, \hat{\mathbf{Z}}_{0}=-\mathbf{A}_{0}^{T} \mathbf{A}^{-1} \hat{\mathbf{L}}+\hat{\mathbf{L}}_{0}, \\
& \mathbf{d}_{\sigma 0}=\mathbf{a}_{\sigma} \mathbf{A}^{-1} \mathbf{A}_{0}-\mathbf{a}_{\sigma 0}, \mathbf{d}_{\sigma \sigma}=-\mathbf{a}_{\sigma} \mathbf{A}^{-1} \mathbf{a}_{\sigma}+\mathbf{a}_{\sigma \sigma}, \hat{\boldsymbol{\sigma}}=-\mathbf{a}_{\sigma} \mathbf{A}^{-1} \hat{\mathbf{L}}+\hat{\mathbf{l}}_{\sigma}
\end{aligned}
$$

Equation (24a) and (24b) are two elasticity equations referring to each bem-elements. They relate the generalized (or weighted) displacements and tractions $\mathbf{Z}_{0}$ defined on the interface boundary $\Gamma_{0}$ and the stresses $\sigma$ at the Gauss point of the bem-elements domain to the interface mechanical and kinematical nodal quantities $\mathbf{X}_{0}$, to the plastic strains $\mathbf{p}$ and to the load vectors $\hat{\mathbf{Z}}_{0}$ and $\hat{\boldsymbol{\sigma}}$. Moreover $\mathbf{D}_{00}, \mathbf{D}_{0 \sigma}, \mathbf{d}_{\sigma 0}, \mathbf{d}_{\sigma \sigma}$ are appropriate stiffness matrices of the bem-elements.

We start by subdividing the body into $m$ bem-elements and by considering Equation (24a) and (24b) for each of these elements. Thus, we obtain two global relations related to all the bem-elements considered, that is,

$$
\begin{aligned}
& \left|\begin{array}{c}
\mathbf{Z}_{0}^{l} \\
\vdots \\
\mathbf{Z}_{0}^{m}
\end{array}\right|=\left|\begin{array}{ccc}
\mathbf{D}_{00}^{l} & \ldots & \mathbf{0} \\
\vdots & \ddots & \vdots \\
\mathbf{0} & \ldots & \mathbf{D}_{00}^{m}
\end{array}\right|\left|\begin{array}{|}
\mathbf{X}_{0}^{l} \\
\vdots \\
\mathbf{X}_{0}^{m}
\end{array}\right|+\left|\begin{array}{ccc}
\mathbf{D}_{0 \sigma}^{l} & \ldots & \mathbf{0} \\
\vdots & \ddots & \vdots \\
\mathbf{0} & \ldots & \mathbf{D}_{0 \sigma}^{m} \\
\mathbf{\sigma}^{m}
\end{array}\right| \begin{array}{|c}
\mathbf{p}^{l} \\
\vdots \\
\mathbf{p}^{m}
\end{array}|+\beta| \begin{array}{c}
\hat{\mathbf{Z}}_{0}^{l} \\
\vdots \\
\hat{\mathbf{Z}}_{0}^{m}
\end{array} \mid \\
& \left|\begin{array}{c}
\boldsymbol{\sigma}^{l} \\
\vdots \\
\boldsymbol{\sigma}^{m}
\end{array}\right|=\left|\begin{array}{ccc}
\mathbf{d}_{\sigma 0}^{l} & \ldots & \mathbf{0} \\
\vdots & \ddots & \vdots \\
\mathbf{0} & \ldots & \mathbf{d}_{\sigma 0}^{m}
\end{array}\right|\left|\begin{array}{|}
\mathbf{X}_{0}^{l} \\
\vdots \\
\mathbf{X}_{0}^{m}
\end{array}\right|+\left|\begin{array}{ccc}
\mathbf{d}_{\sigma \sigma}^{l} & \ldots & \mathbf{0} \\
\vdots & \ddots & \vdots \\
\mathbf{0} & \ldots & \mathbf{d}_{\sigma \sigma}^{m} \\
\vdots \\
\mathbf{p}^{m}
\end{array}\right|+\beta\left|\begin{array}{|c}
\mathbf{p}^{l} \\
\vdots \\
\hat{\boldsymbol{\sigma}}^{m}
\end{array}\right|
\end{aligned}
$$

or in compact form

$$
\begin{aligned}
\mathbf{Z}_{0} & =\mathbf{D}_{00} \mathbf{X}_{0}+\mathbf{D}_{0 \sigma} \mathbf{p}+\beta \hat{\mathbf{Z}}_{0} \\
\boldsymbol{\sigma} & =\mathbf{d}_{\sigma 0} \mathbf{X}_{0}+\mathbf{d}_{\sigma \sigma} \mathbf{p}+\beta \hat{\boldsymbol{\sigma}}
\end{aligned}
$$

formally equal to Equation (24a) and (24b), but regarding all the bem-elements in global form.

We introduce the nodal interface vector $\zeta_{0}$ of the mechanical and kinematical unknowns related to the assembled system and perform a suitable nodal variable condensation through the matrices of equilibrium $\mathbf{L}^{T}$ and of compatibility $\mathbf{N}$, respectively:

$$
\left|\begin{array}{c}
\mathbf{F}_{0}^{l} \\
-\mathbf{U}_{0}^{l} \\
\hline \vdots \\
\vdots \\
\hline \mathbf{F}_{0}^{m} \\
-\mathbf{U}_{0}^{m}
\end{array}\right|=\left|\begin{array}{cc}
\left(\mathbf{L}^{l}\right)^{T} & \mathbf{0} \\
\mathbf{0} & \mathbf{N}^{l} \\
\vdots & \vdots \\
\vdots & \vdots \\
\hdashline & \multicolumn{1}{|c}{\mathbf{F}_{0}} \\
\left.\hdashline \mathbf{L}^{m}\right)^{T} & \mathbf{0} \\
\mathbf{0} & \mathbf{N}^{m}
\end{array}\right| \text { i.e. } \mathbf{X}_{0}=\mathbf{E} \boldsymbol{U}_{0}
$$

The latter relation has to be considered as a strong regularity condition, that is,

$$
\begin{aligned}
\mathbf{F}_{0}^{j} & =-\mathbf{F}_{0}^{j+1}=\mathbf{F}_{0} \\
-\mathbf{U}_{0}^{j} & =-\mathbf{U}_{0}^{j+1}=-\mathbf{U}_{0}
\end{aligned}
$$

with $j=1, \ldots, m$ the generic bem-elements, $\left(\mathbf{L}^{j}\right)^{T}$ being full by $1,-1$ and 0 , and $\mathbf{N}^{j}$ full by 1 and 0 .

The same transposed matrices $\mathbf{L}^{j}$ and $\mathbf{N}^{j T}$ define the weighted equilibrium and compatibility, respectively.

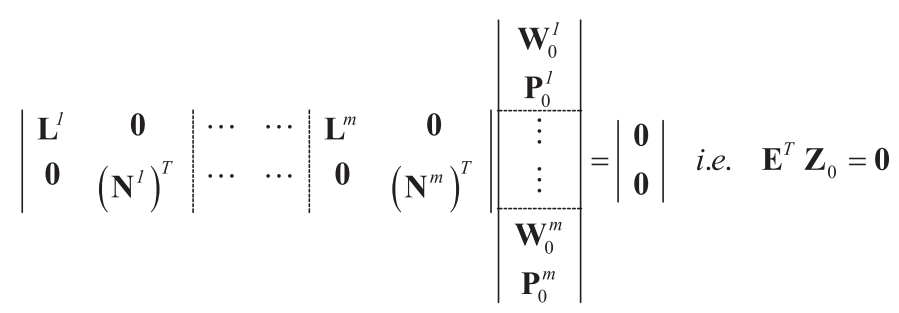


The latter relation has to be considered as a weak regularity condition, that is,

$$
\begin{aligned}
& \mathbf{W}_{0}^{j}=\mathbf{W}_{0}^{j+1} \\
& \mathbf{P}_{0}^{j}=-\mathbf{P}_{0}^{j+1}
\end{aligned}
$$

Equations (29) and (31) have to be considered respectively as strong and weak coupling conditions of Equations (3a) and ( $3 b$ ) for a multidomain SGBEM problem.

The compact forms of Equations (28) and (30) introduced in Equation (27a) and (27b) give rise to the following relations:

$$
\begin{aligned}
& \mathbf{K}_{00} \zeta_{0}+\mathbf{K}_{0 \sigma} \mathbf{p}+\beta \hat{\mathbf{f}}_{0}=\mathbf{0} \\
& \boldsymbol{\sigma}=\mathbf{k}_{\sigma 0} \zeta_{0}+\mathbf{k}_{\sigma \sigma} \mathbf{p}+\beta \hat{\boldsymbol{\sigma}}
\end{aligned}
$$

where the following positions are valid:

$$
\mathbf{K}_{00}=\mathbf{E}^{T} \mathbf{D}_{00} \mathbf{E}, \mathbf{K}_{0 \sigma}=\mathbf{E}^{T} \mathbf{D}_{0 \sigma}, \hat{\mathbf{f}}_{0}=\mathbf{E}^{T} \hat{\mathbf{Z}}_{0}, \mathbf{k}_{\sigma 0}=\mathbf{d}_{\sigma 0} \mathbf{E}, \mathbf{k}_{\sigma \sigma}=\mathbf{d}_{\sigma \sigma}
$$

Introducing a new variable condensation through the replacement of the $\zeta_{0}$ vector extracted from Equation (32a) into Equation (32b), the self-equilibrium stress equation is obtained:

$$
\boldsymbol{\sigma}=\mathbf{K} \mathbf{p}+\beta \hat{\boldsymbol{\sigma}}_{s}
$$

where

$$
\mathbf{K}=-\mathbf{k}_{\sigma 0} \mathbf{K}_{00}^{-1} \mathbf{K}_{0 \sigma}+\mathbf{k}_{\sigma \sigma}, \hat{\boldsymbol{\sigma}}_{s}=-\mathbf{k}_{\sigma 0} \mathbf{K}_{00}^{-1} \hat{\mathbf{f}}_{0}+\hat{\boldsymbol{\sigma}}
$$

Equation (34) provides the stress at the Gauss points of each bem-elements as a function of the plastic strains $\mathbf{p}$ and of the external actions $\hat{\boldsymbol{\sigma}}_{s}$, the latter amplified by $\beta$. $\mathbf{K}$ is defined as a self-stress influence matrix, fully populated, not symmetric and semidefined negative $[8,9]$. The evaluation of this matrix only involves the elastic characteristic of the material and the structure geometry.

Briefly, the three elastic equations obtained inside the multidomain SGBEM formulation able to provide the solution of the elastoplasticity-contact/detachment problem are the following:

$$
\begin{aligned}
\mathbf{X} & =-\mathbf{A}^{-1}\left(\mathbf{R}_{0 \sigma} \mathbf{p}+\beta \hat{\mathbf{f}}_{x}\right) \\
\zeta_{0} & =-\mathbf{K}_{00}^{-1}\left(\mathbf{K}_{0 \sigma} \mathbf{p}+\beta \hat{\mathbf{f}}_{0}\right) \\
\boldsymbol{\sigma} & =\mathbf{K} \mathbf{p}+\beta \hat{\boldsymbol{\sigma}}_{s}
\end{aligned}
$$

where Equation (36a) is obtained utilizing the condensation (28), and the vector $\zeta_{0}$ given by (36b) in Equation (22a).

In Equation (36a), the following definitions are valid:

$$
\mathbf{R}_{0 \sigma}=-\mathbf{A}_{0} \mathbf{E} \mathbf{K}_{00}^{-1} \mathbf{K}_{0 \sigma}+\mathbf{A}_{\sigma}, \hat{\mathbf{f}}_{x}=-\mathbf{A}_{0} \mathbf{E} \mathbf{K}_{00}^{-1} \hat{\mathbf{f}}_{0}+\hat{\mathbf{L}}
$$

All Equation (36a)-(36c) are exclusively functions of the plastic strains $\mathbf{p}$ stored during the loading/unloading process and of the load multiplier $\beta$.

A characteristic aspect of the present formulation consists in the separate use of these equations to obtain the solution of the elastoplasticity-contact/detachment problem through an iterative approach.

Summarizing:

- Equation (36a) is utilized to solve the contact problem: indeed, for each substructure, the vector $\mathbf{X}=\left|\begin{array}{ll}\mathbf{F}_{1} & -\mathbf{U}_{2}\end{array}\right|^{T}$ collects the nodal forces $\mathbf{F}_{1}$ of the constrained boundary $\Gamma_{1}$ and the nodal displacements $-\mathbf{U}_{2}$, changed in sign, of the free boundary $\Gamma_{2}$, the latter being the check quantities governing the contact problem, when these are compared with the other displacements $-\mathbf{U}_{2}$ of the contiguous substructure.

- Equation (36b) is utilized to solve the detachment problem: indeed, the vector $\zeta_{0}=$ $\left|\begin{array}{ll}\mathbf{F}_{0} & -\mathbf{U}_{0}\end{array}\right|^{T}$ collects the nodal disclacements $-\mathbf{U}_{0}$, changed in sign, of the interface boundary $\Gamma_{0}$ and the nodal forces $\mathbf{F}_{0}$ of the same boundary, the latter being the check quantities governing the detachment problem. 
- Lastly, Equation (36c), called the self-stress equation, is utilized inside the elastoplasticity field.

The use of Equation (36), characterizing a geometrical and physical nonlinear problem, is able to provide the full solution of the elastoplasticity-contact/detachment problem, following an approach such as an incremental strategy. In the following subsections, the algorithms and the strategies utilized in solving the subproblems of contact/detachment, through the use of the discrete Signorini conditions, and the elastoplasticity subproblem, through a predictor-corrector strategy for active macro-zones, are shown.

\subsection{Incremental contact/detachment via multidomain symmetric Galerkin BEM}

Inside the SGBEM topic, to reach the analytical solution to this frictionless contact-detachment problem, an iterative LCP procedure can be employed once the elastic analysis has been performed using Equation (36a) and (36b).

For this purpose, it has to be remembered that the unknown vectors $\mathbf{F}_{0}, \mathbf{U}_{2}^{A}$ and $\mathbf{U}_{2}^{B}$ are referred to the nodes of the in-contact boundary and to the nodes of the detached one. These check quantities can be obtained by Equation (36a) and (36b). The vector $\mathbf{F}_{0}$ represents the nodal forces of the body $A$, computed in the boundary zone $\Gamma_{0}$, and the vectors $\mathbf{U}_{2}^{A}$ and $\mathbf{U}_{2}^{B}$ represent the nodal displacements of the boundaries of $\Gamma_{2}^{A}$ and $\Gamma_{2}^{B}$.

With reference to the system of the two in-contact bodies, whose boundaries are discretized into boundary elements, Equation (4a)-(4d) characterizing the boundary conditions of the phenomenon being examined can be rewritten in a very similar way. Indeed, the nodal boundary vectors $\mathbf{F}_{0}=\mathbf{F}_{0}^{A}=-\mathbf{F}_{0}^{B},\left(-\mathbf{U}_{2}^{A}\right)$ and $\left(-\mathbf{U}_{2}^{B}\right)$, changed in sign, must substitute the vectors $\mathbf{t}_{0}^{A}, \mathbf{u}_{2}^{A}$ and $\mathbf{u}_{2}^{B}$, the latter being defined at each point on the boundary. Therefore,

$$
\begin{gathered}
\mathbf{N}_{2}^{A}\left(\left(\mathbf{U}_{2}^{A}-\mathbf{U}_{2}^{B}\right)-\mathbf{H}\right) \leqslant \mathbf{0}, \quad \mathbf{C}=\mathbf{0} \text { gap condition } \\
\mathbf{N}_{0}^{A} \mathbf{F}_{0}-\mathbf{C} \leqslant \mathbf{0}, \quad \mathbf{H}=\mathbf{0} \text { contact condition } \\
{\left[\mathbf{N}_{2}^{A}\left(\left(\mathbf{U}_{2}^{A}-\mathbf{U}_{2}^{B}\right)-\mathbf{H}\right)\right]\left[\mathbf{N}_{2}^{A} \mathbf{F}_{2}\right]=0 \text { complementarity condition on } \Gamma_{2}} \\
{\left[\mathbf{N}_{0}^{A}\left(\mathbf{U}_{0}^{A}-\mathbf{U}_{0}^{B}\right)\right]\left[\mathbf{N}_{0}^{A} \mathbf{F}_{0}-\mathbf{C}\right]=0 \text { complementarity condition on } \Gamma_{0}}
\end{gathered}
$$

with $\mathbf{N}_{0}^{A}$ and $\mathbf{N}_{2}^{A}$ collecting the transposes of the normal vectors associated with the boundary nodes of the contact elements and of the detached elements, respectively.

All the mechanical and kinematical nodal quantities are referred to the load increment $(n+1)$.

The vector $\mathbf{H}$ collects all the weighted nodal gaps between the corresponding sides of the boundaries $\Gamma_{2}^{A}$ and $\Gamma_{2}^{B}$ considered, in the zone of potential contact, whereas the vector $\mathbf{C}$ collects the weighted cohesion between the sides, which are in contact, in the zone of potential detachment $\Gamma_{0}$.

\subsection{Incremental elastoplastic analysis via multidomain symmetric Galerkin BEM}

A brief description of the strategy utilized for incremental elastoplastic analysis via multidomain SGBEM, called elastoplastic macro-zone analysis, is provided in this section. The complete version can be found in [8]. For each loading step and at each bem-elements, this analysis uses Equation (34) both to evaluate the trial stresses in the predictor phase and to compute the plastic strains in the corrector phase.

Let us start by evaluating the trial stresses, that is, the purely elastic response at the load step $n+1$ in each of $m$ bem-elements of the discretized body. For this purpose Equation (34) provides all the predictors $\boldsymbol{\sigma}_{(n+1)}^{*}$ as functions of the plastic strain vector $\mathbf{p}_{(n)}$, stored at step $n$, and of all the increments $\Delta \mathbf{p}$ inside step $n+1$, and of the external load $\hat{\boldsymbol{\sigma}}_{s}$, amplified by $\beta_{(n+1)}$ :

$$
\boldsymbol{\sigma}_{(n+1)}^{*}=\mathbf{K} \mathbf{p}_{(n+1)}+\beta_{(n+1)} \hat{\boldsymbol{\sigma}}_{s} \text { with } \mathbf{p}_{(n+1)}=\mathbf{p}_{(n)}+\Delta \mathbf{p}
$$

where $\beta_{(n+1)}=\beta_{(n)}+\Delta \beta$ is the load factor, and the $\mathbf{K}$ matrix is fully populated and regards all the bem-elements, the locus of possible nonlinear phenomena, obtained through domain discretization. 
A check on the plastic consistency condition of the stresses, computed at appropriately chosen points inside each bem-elements, is performed using the yield condition expressed in this context through the von Mises law, that is,

$$
F\left[\boldsymbol{\sigma}_{i(n+1)}^{*}\right]=\frac{1}{2} \boldsymbol{\sigma}_{i(n+1)}^{* T} \boldsymbol{M} \boldsymbol{\sigma}_{i(n+1)}^{*}-\sigma_{y}^{2} \leqslant 0 \text { with } i=1 \ldots m
$$

where $\boldsymbol{M}$ is a matrix of constants, and $\sigma_{y}$ is the uniaxial yield stress. In the $a$ bem-elements (with $a \leqslant m$ ) where this inequality is violated, a return mapping phase occurs in order to evaluate the plastic strains and the direction of the plastic flow.

This phase, called the corrector phase, uses the first term of Equation (34) to obtain the elastoplastic solution at every bem-elements where the plastic consistency condition is violated. In this phase, the vector $\sigma$, representing the end-step stress, and the increment of the volumetric plastic strain vector $\Delta \mathbf{p}$, are unknown quantities. The latter are the plastic strains to be imposed at every plastically active bem-elements in order to obtain the stress on the yield boundary of the elastic domain, characterized by the direction of the plastic flow. Obviously, inside each loading step, the macro-zone elastoplastic analysis requires the corrector phase to be repeated until all the predictors satisfy the plastic consistency conditions.

In detail, for each active $h$ bem-element $(h=1, \ldots, a)$, the elastoplastic algorithm allows one to write a nonlocal system at the $n+1$ load step simultaneously in all the plastically active bem-elements, defining the macro-zones, identified in the previous predictor phase, that is,

$$
\begin{gathered}
\boldsymbol{\sigma}_{a(n+1)}=\boldsymbol{\sigma}_{a(n+1)}^{*}+\mathbf{K}_{a a} \Delta \mathbf{p}_{a(n+1)} \\
F\left[\boldsymbol{\sigma}_{a(n+1)}\right] \leqslant \mathbf{0}, \quad \Delta \boldsymbol{\Lambda}_{a(n+1)} \geqslant \mathbf{0}, \quad \Delta \boldsymbol{\Lambda}_{a(n+1)} \boldsymbol{F}\left[\boldsymbol{\sigma}_{a(n+1)}\right]=\mathbf{0}
\end{gathered}
$$

where Equation (42a)-(42c) are the plastic admissibility conditions for the $a$ bem-elements.

The index $a$ characterizes vectors and matrices connecting the mechanical and kinematical quantities relating to all the active $a$ bem-elements. The $\mathbf{K}_{a a}$ matrix coefficients are derived from the $\mathbf{K}$ matrix present in Equation (34), by extracting the related blocks. Obviously this submatrix, and therefore its dimension, is subjected to change, for every load step.

In the following equations, the subscript $(n+1)$ has been omitted for convenience.

In the hypothesis that, for each $h$-th bem-elements, the shape function defined in Equation $(17 \mathrm{~g})$ regarding the plastic strain modeling is the same as the shape function relating to the plastic multiplier, that is, $\Delta \lambda_{h}=\psi_{p} \Delta \Lambda_{h}$ with $\psi_{p} \geqslant 0$, the plastic strain increment for the $a$-th active bem-elements is expressed as

$$
\Delta \mathbf{p}_{a}=\partial_{\boldsymbol{\sigma}_{a}} \mathbf{F}\left[\boldsymbol{\sigma}_{a}\right] \Delta \boldsymbol{\Lambda}_{a}=\mathbf{M}_{a} \boldsymbol{\sigma}_{a} \Delta \boldsymbol{\Lambda}_{a}
$$

where the product $\mathbf{M}_{a} \boldsymbol{\sigma}_{a}$ is a diagonal matrix ( $3 a \mathrm{x} a$ ), each diagonal term being made by a vector $(3 \times 1)$ and the vector $\Delta \boldsymbol{\Lambda}_{a}$ collecting all the plastic strain intensities of the plastically active bem-elements.

The solving nonlinear system for all the active $a$ bem-elements is the following:

$$
\left\{\begin{array}{l}
\boldsymbol{\sigma}_{a}-\boldsymbol{\sigma}_{a}^{*}-\mathbf{K}_{a a} \mathbf{M}_{a} \boldsymbol{\sigma}_{a} \Delta \mathbf{\Lambda}_{a}=\mathbf{0} \\
F\left[\boldsymbol{\sigma}_{a}\right]=\mathbf{0}
\end{array}\right.
$$

or, in explicit form, using the von Mises yield law and the plastic flow role given by Equation (43):

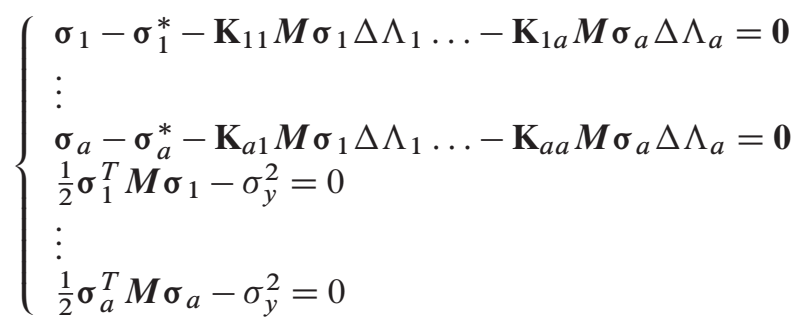


where $\sigma_{a}$ is the stress solution located on the yield surface of the elastic domain of all the active bemelements, $\boldsymbol{\sigma}_{a}^{*}$ is the elastic predictor, and $\mathbf{K}_{a a} \mathbf{M}_{a} \boldsymbol{\sigma}_{a} \Delta \boldsymbol{\Lambda}_{a}$ is the corrective components (containing local and nonlocal contributions).

The approximate solution of this nonlinear problem involving all the plastically active bemelements, in terms of $\boldsymbol{\sigma}_{a}$ and $\Delta \boldsymbol{\Lambda}_{a}$, can be obtained by applying the Newton-Raphson procedure. The existence of the solution of the nonlinear problem (45) is guaranteed unless plastic collapse takes place. In Figure 2, the elastoplastic-contact/detachment procedure is shown. The strategy proposed in this paper proves to be computationally onerous because it is based on evaluation of the solution to the elastoplasticity-contact/detachment problem following an iterative approach, but shows great efficiency and strength and suffers very little from numerical instability problems. In addition, the symmetric BEM quite significantly reduces the calculus time in comparison with other formulations based on integral equations because of the closed-form evaluation of all the system coefficients and, compared with the finite element method (FEM), because of the partial domain discretization.

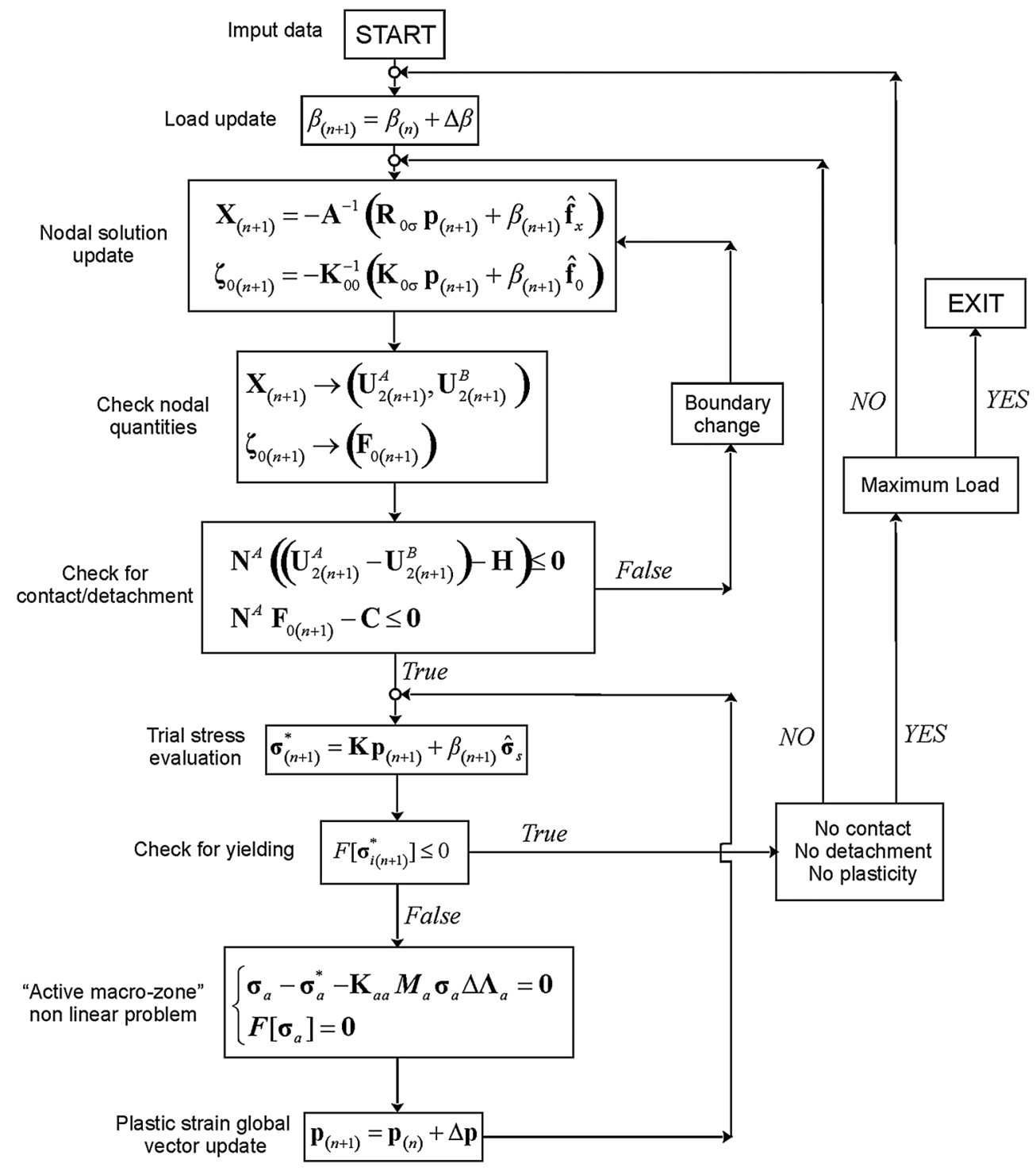

Figure 2. Flow chart of the elastoplastic-contact/detachment procedure. 


\section{NUMERICAL RESULTS}

In order to show the efficiency of the proposed method some numerical tests were performed under the following hypotheses: bidimensional bodies, frictionless and slidingless contact, plane strain condition, perfect plasticity, von Mises law and associated plastic flow rule. The analyses of the two tests were performed using the Karnak program [32].

\subsection{Alloy beam supported on two steel blocks}

In this subsection, an alloy beam having a box section, supported on two steel blocks, is shown.

This beam is subjected to a distributed load at the top having an initial value $q=100 \mathrm{daN} / \mathrm{m}$. In Figure 3, where all the dimensions are expressed in millimeter, the physical and geometrical characteristics of the structural system are shown.

The presence of the roller as a constraint at the basement of the steel block avoids the rise of tangential stresses in the support zone of the beam. As a consequence, the structural problem may be considered as a frictionless and slide-free model. The cohesion value is assumed to be $c=0$.

In Figure 4, the discretization utilized is shown in detail:

- The steel support is elastic during all the load process, characterized by only one macroelement having a boundary discretization into elements with constant length $p=50 \mathrm{~mm}$, with the exception of the contact zone with the beam where the boundary element length was assumed to be $p=4 \mathrm{~mm}$.

- The alloy beam has elastoplastic behavior characterized by the following: discretization of the domain through four macroelement (three elements regarding the upper and lower bases of the beam and one element regarding the stem) only having elastic behavior, and 132 bem-elements, of which 72 near the support and 60 in the middle of the beam having elastoplastic behavior.

- Discretization of the boundary into elements with constant length $p=50 \mathrm{~mm}$, with the exception of the contact zone with the beam where the same discretization of the support was assumed.

The elastoplastic-contact/detachment analysis was performed for this structure having as its final response a detachment length between the alloy beam and the steel support $d=196.1 \mathrm{~mm}$, which remains unchanged during all the load process.

In Figures 4(b) and (c), the active bem-elements are shown in grey at plastic collapse: these bem-elements characterize two large hinges representing the cause of the collapse mechanism. In Figure 4(d), the strain shape at plastic collapse, obtained by high amplification of the displacements, is shown.

Figure 5 shows the load-displacement characteristic curve through which the value of the collapse load $q=884 \mathrm{daN} / \mathrm{m}$ was obtained.

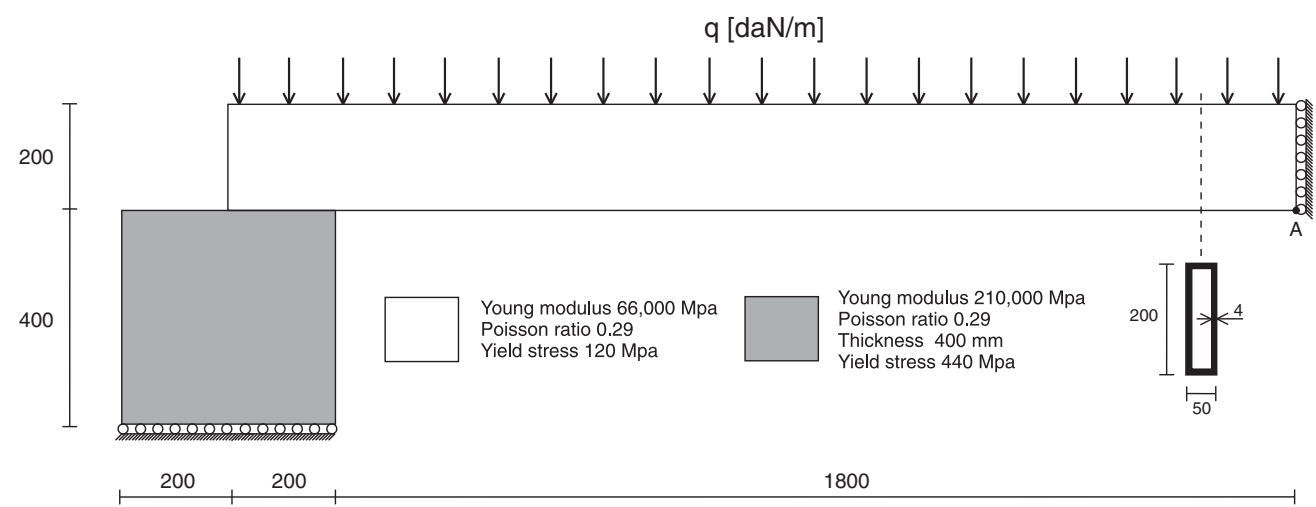

Figure 3. Alloy beam supported on two steel blocks. Geometric description. 


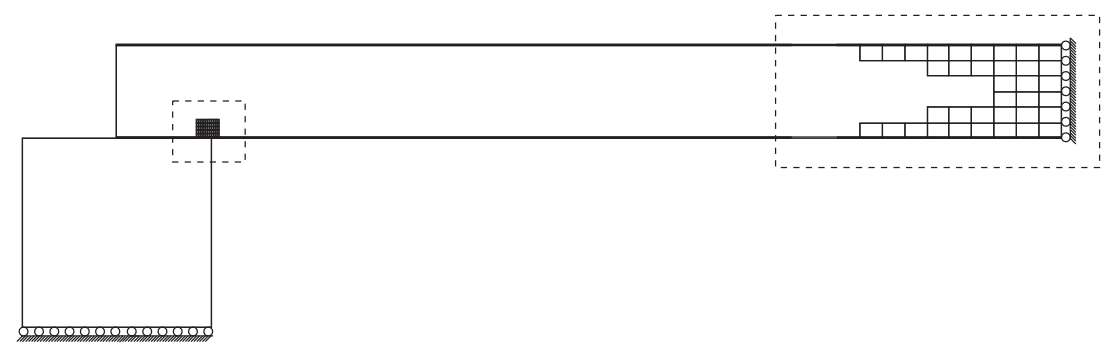

(a)

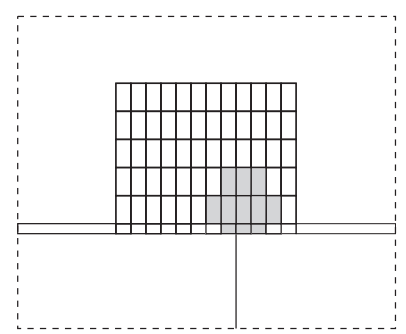

(b)

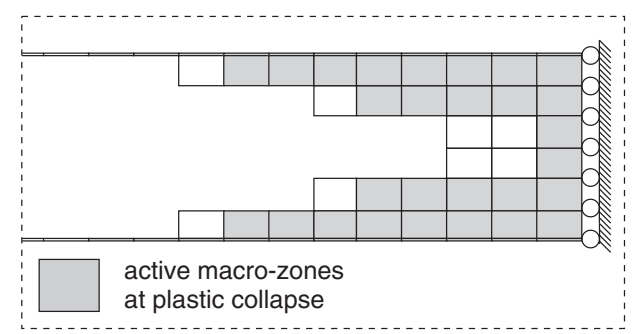

(c)

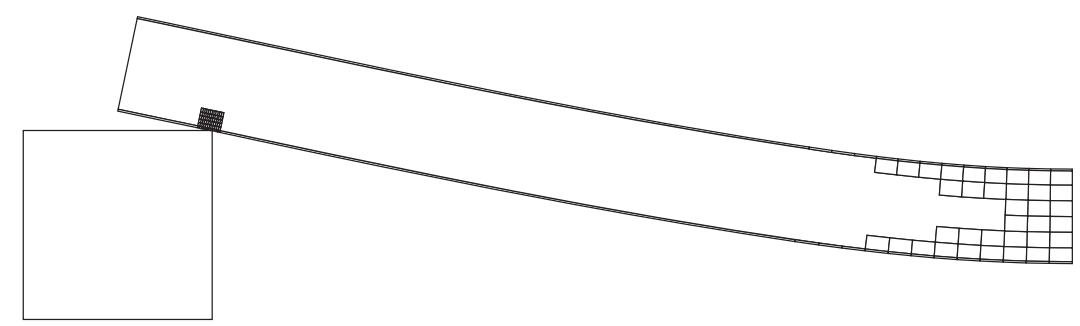

(d)

Figure 4. (a) Discretization adopted, (b) and (c) active macro-zones at plastic collapse and (d) strain shape at plastic collapse.

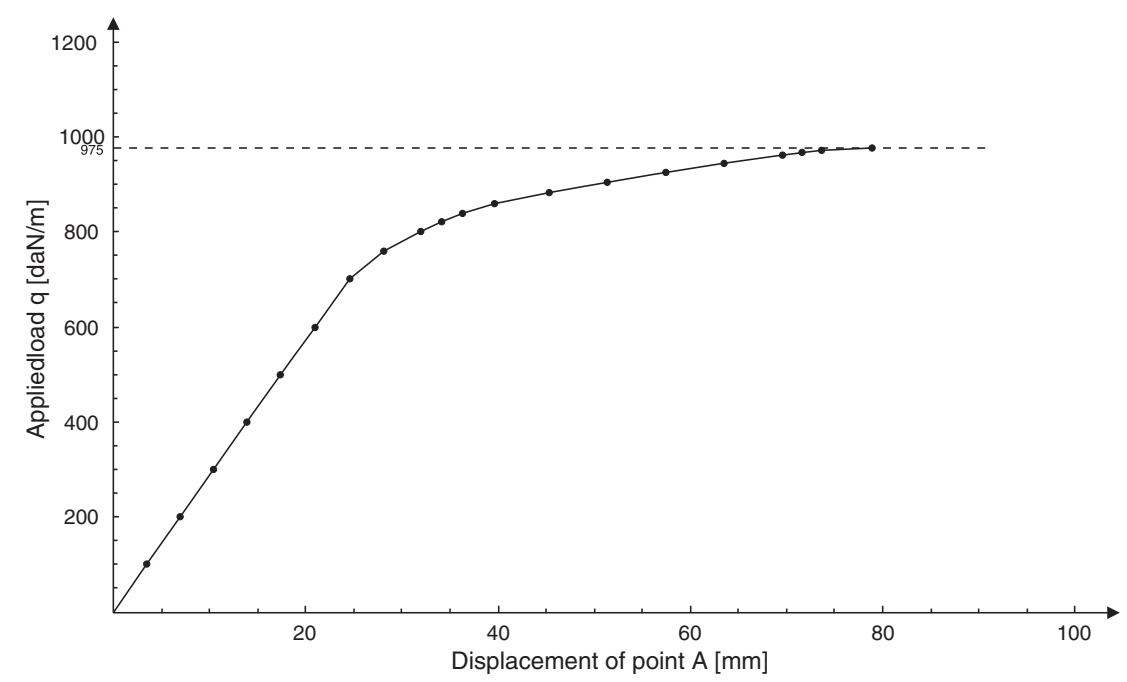

Figure 5. Load-displacement characteristic curve.

In Figure 6 the horizontal stress distribution $\sigma_{x}$ near the middle section is shown for increasing load values up to the load value before collapse. It is to be noted that when the collapse load is reached, the plastic zone involves the bases of the beam and a large part of the stem, whereas the 


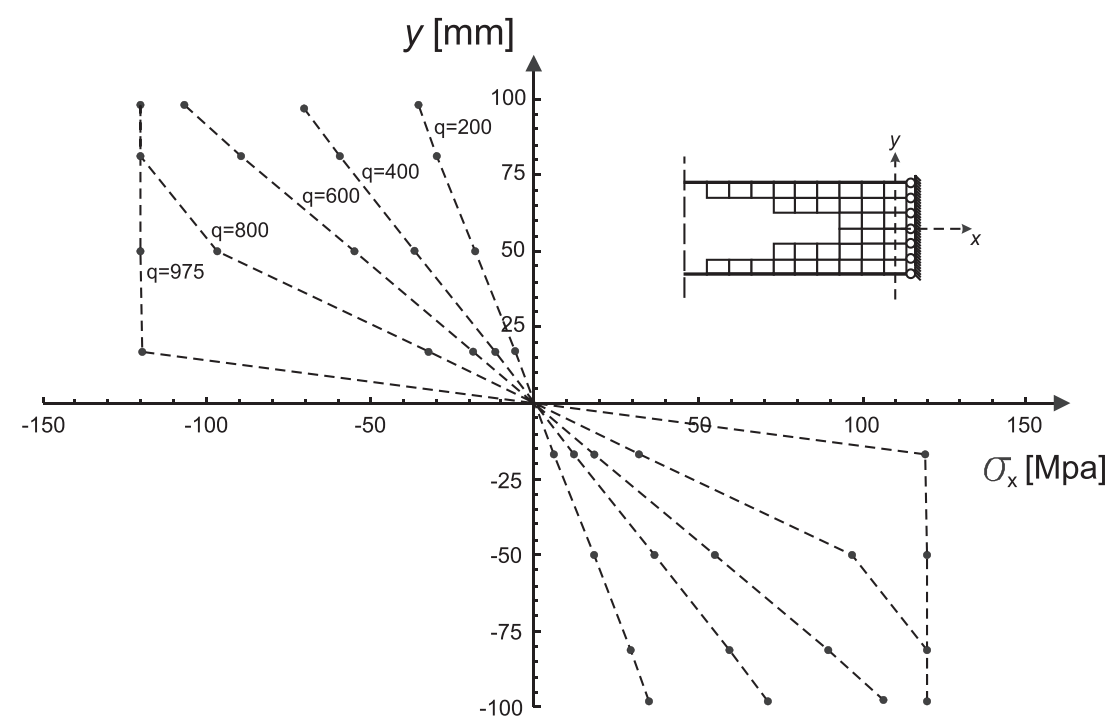

Figure 6. Stress distributions $\sigma_{x}$ during the load process.
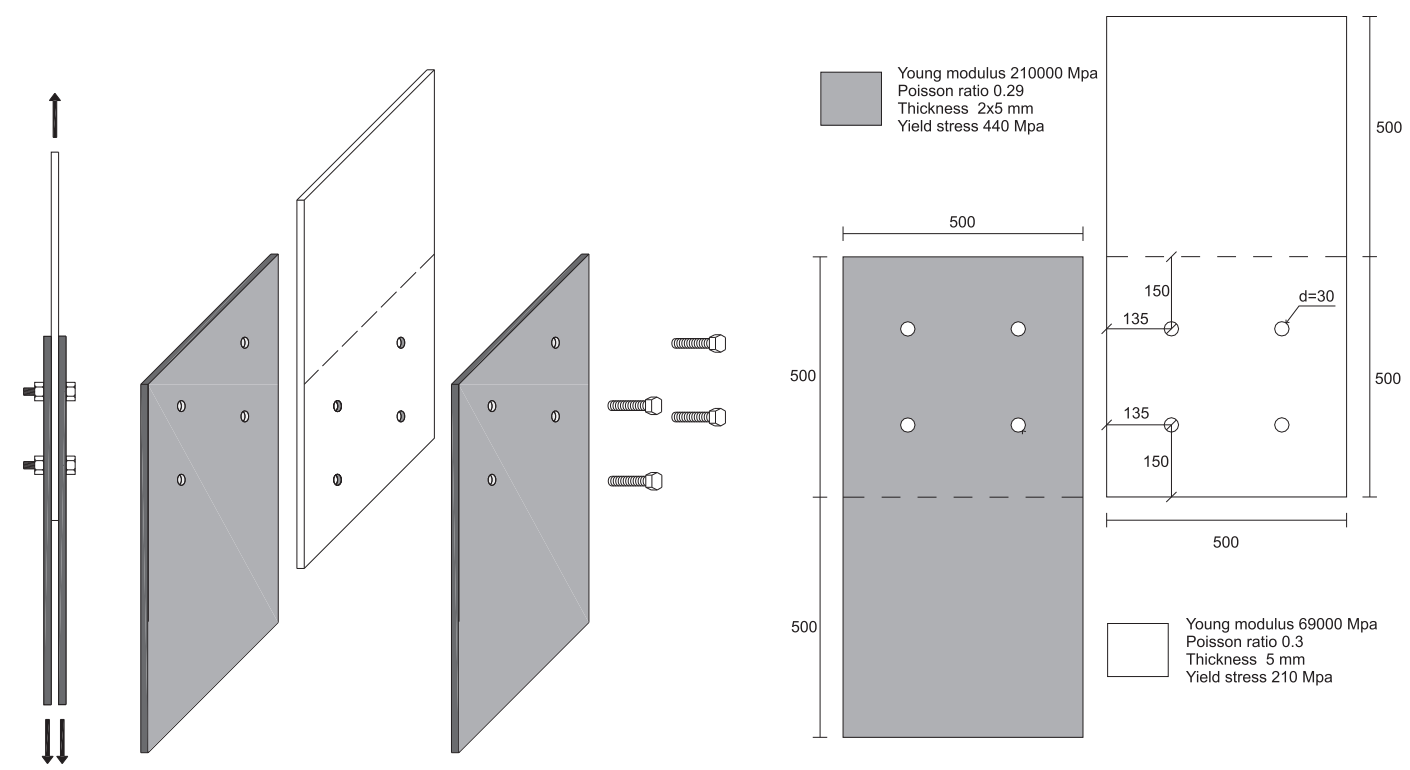

Figure 7. Nonhomogeneous system of plates connected by bolts. Geometric description.

elastic zone only regards the central part of the section. In addition, plasticity reaches 11 bem-elements located on each base of the beam.

\subsection{Nonhomogeneous system of plates connected by bolts}

A system constituted by plates, having different characteristics, connected to one another, is shown in Figure 7. An alloy plate is connected to two steel plates through four bolts. The system is subjected to traction forces distributed on the upper extremity of the alloy plate, whereas the steel plates are constrained on the lower extremities. In Figure 7, the physical-geometrical characteristics of the system are shown. The dimensions are expressed in millimeter. 

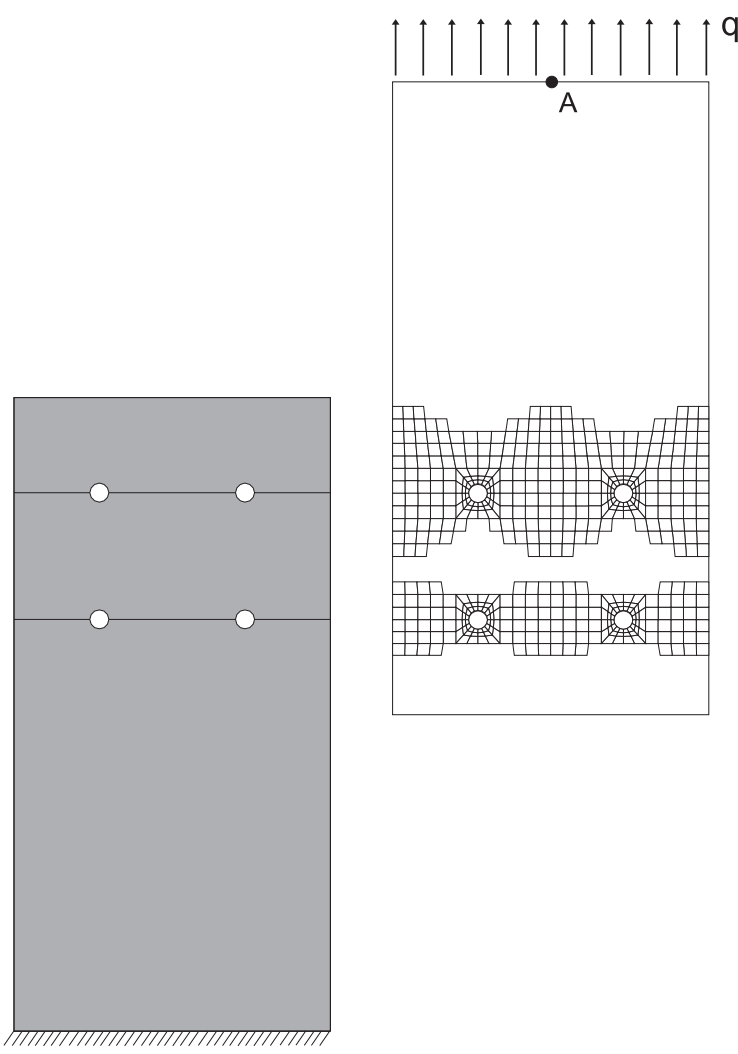

Figure 8. Discretization adopted.

The analysis is performed in the hypothesis of a strain plain state because the steel plates can be considered as a single plate having double thickness. In this way, the alloy plate and the equivalent steel one belong to two different layers. Transfer of stresses from one plate to another is only ensured by four bolts, the latter being supposed to have infinitely rigid behavior during all the loading process. These bolts are connected below the alloy plate and above the steel ones.

Moreover, possible friction between the two plate systems is neglected, making the collapse load lower and therefore, safer.

In Figure 8, the discretization utilized is shown in detail:

- The equivalent steel plate is subdivided into three macroelements having purely elastic behavior; this discretization into three macroelements being necessary to guarantee the uniqueness of the solution, which typically occurs in SGBEM [25,33-35] in the case of systems, which are not multiconnected because of the presence of the holes or openings. In this example, the uniqueness is guaranteed through a domain discretization having the interfaces, which intersect the holes.

- The alloy plate is characterized by a domain discretization into 520 bem-elements inserted to simulate nonlinear physical behavior and three macroelements having purely elastic behavior. The extent of the potentially plastic zones can be defined either through the preliminary help of elastic analysis or refining the mesh in an elastoplastic analysis.

- The four bolts are characterized by four polygonal substuctures supposed to be rigid and having elastic behavior during all the loading process.

Figure 9(a) shows in grey, the macro-zones involved in plastic strain storage immediately before plastic collapse. In Figure 9(b), the load-displacement characteristic curve is shown, computing the displacement at the check point $\mathrm{A}$ at the top of the alloy plate (Figure 8). The curve show that plastic collapse occurs for a load value of $\beta \mathrm{q} / \sigma_{y}=0.8781$. 


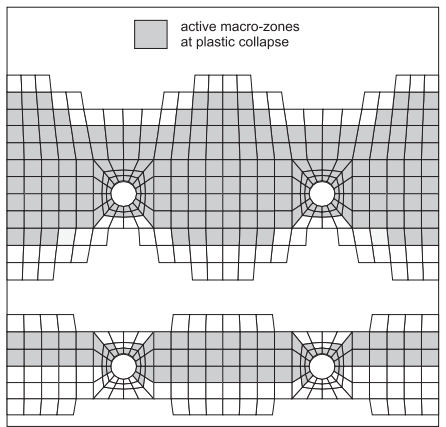

(a)

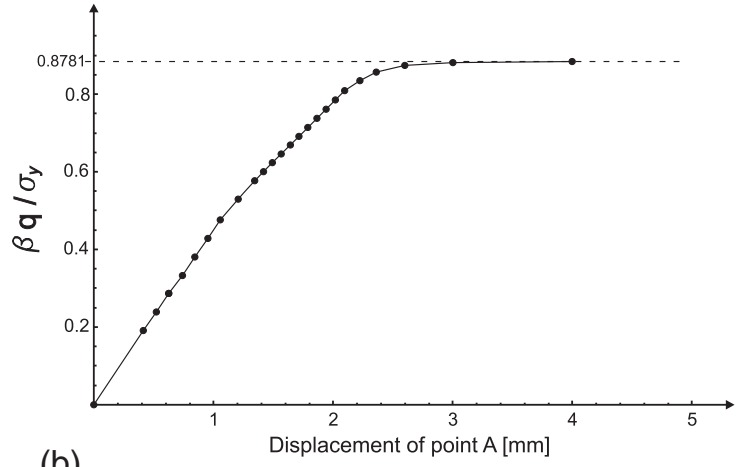

(b)

Figure 9. (a) Active macro-zones at plastic collapse and (b) load-displacement characteristic curve.

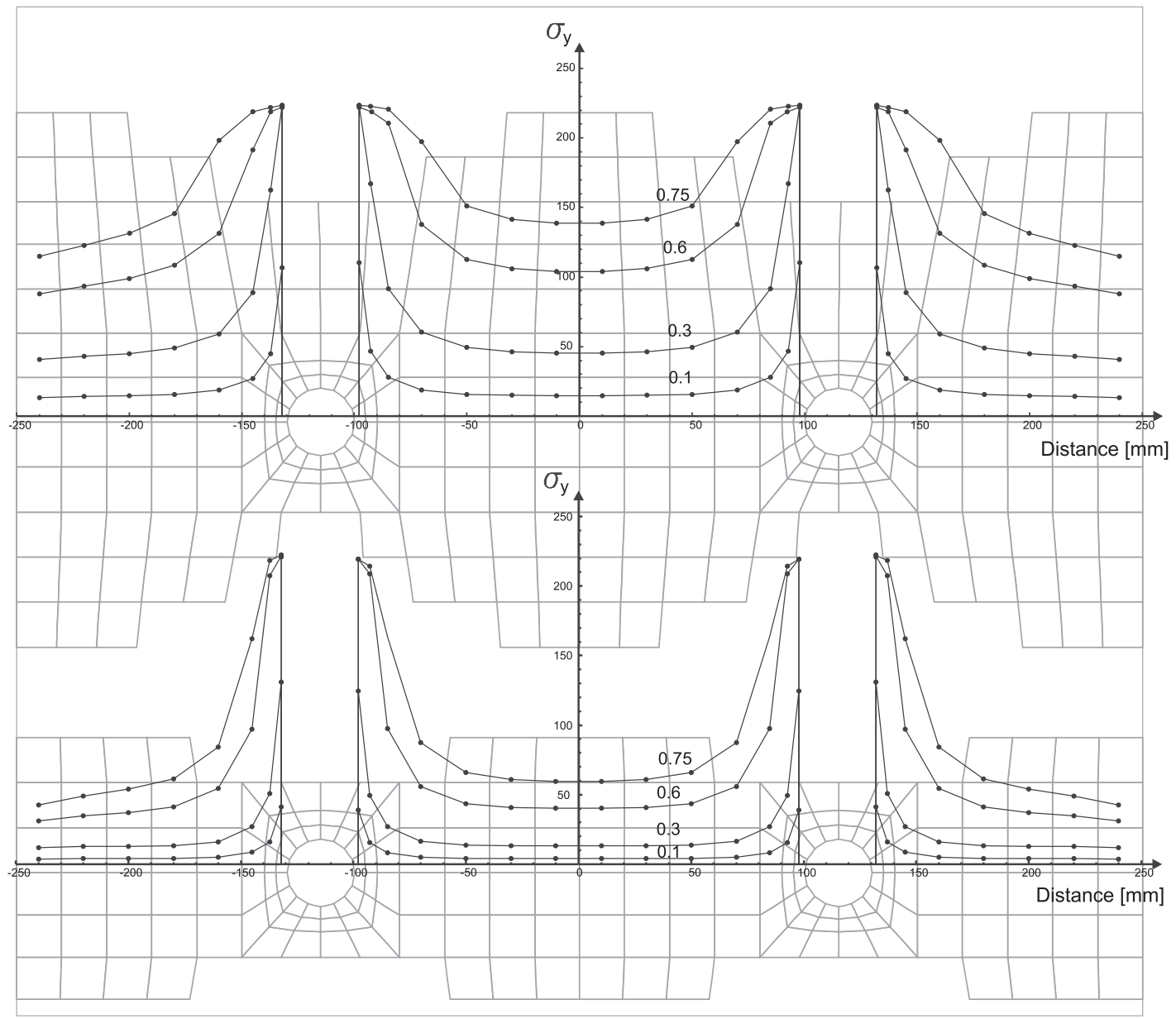

Figure 10. Stress distributions during the load process $\sigma_{y}$.

In Figure 10, the variation in the vertical stress distribution $\sigma_{y}$ along the horizontal sections intercepting the four holes is shown for different load values. It is to be noted that the highest peaks of the stress are located close to the four holes but have higher values in the upper holes as the plastic effect reduces these peaks distributing the stresses in a more uniform way. 
In Figure 11, the other components of stress $\sigma_{x}$ and $\sigma_{x y}$ are shown along the same sections and for the same load process.

In Figure 12(a) the strain shape, for a load value $\beta \mathrm{q} / \sigma_{y}=0.75$ preceding the plastic load and characterizing the shearing effect caused by the 4 bolts, is shown.

The Figure 12(b), the equilibrium is shown in the same load condition: the load value and the four reactions at the contact zones present a very low imbalance, given by $\mathrm{F}_{q y}-\sum_{i=1}^{4} \mathrm{~F}_{i y}=200 \mathrm{daN}$ with a percentage error of $\mathbf{e} \%=0.53$. It is to be noted that when the load increases and the plastic effect mainly regard the upper holes, the greater contribution of the reaction moves to the lower holes.

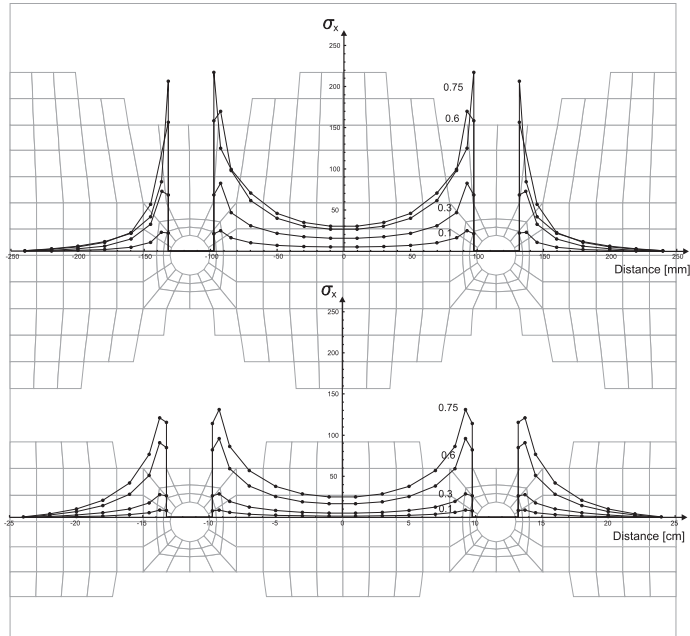

(a)

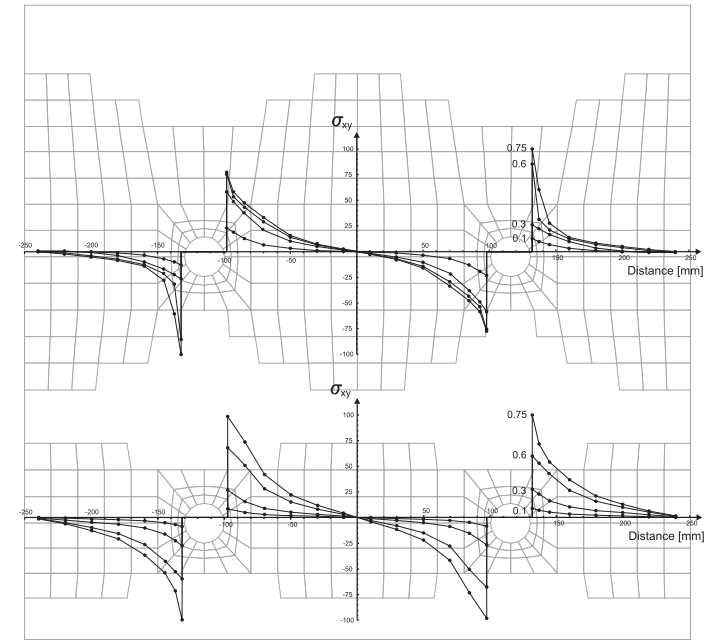

(b)

Figure 11. Stress distributions during the same load process: (a) $\sigma_{x}$ and (b) $\sigma_{x y}$.

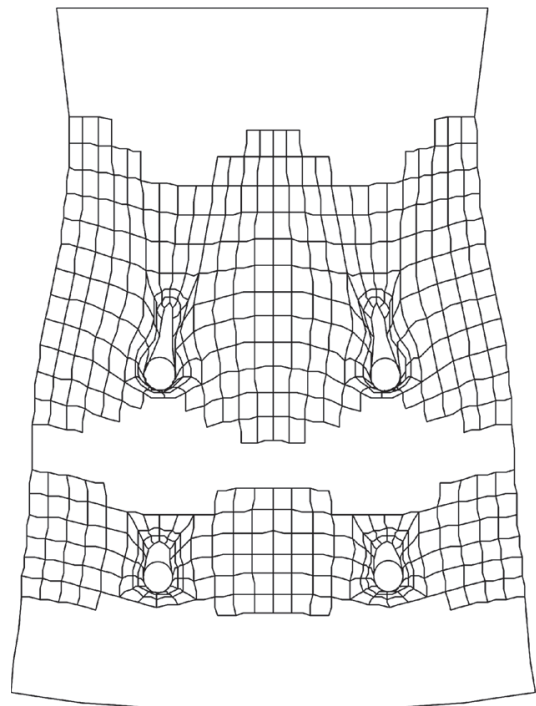

(a)

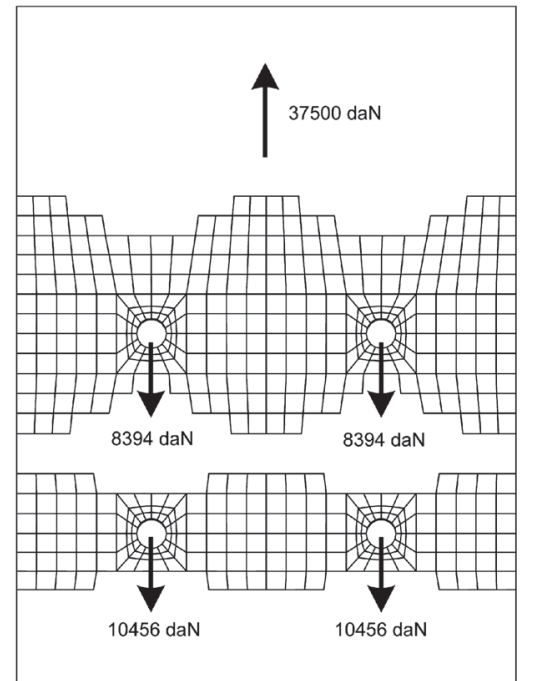

(b)

Figure 12. (a) Strain shape and (b) equilibrium condition before the plastic collapse. 


\section{CONCLUSIONS}

In this paper, a strategy to solve the elastoplastic-contact/detachment problem is shown utilizing the SGBEM inside the multidomain approach. An iterative strategy, based on the LCP, is used to solve both the two subproblems of elastoplasticity and contact/detachment. In detail, the elastoplastic problem utilizes the well-known self-stress matrix whose characteristics made it possible to develop a nonlocal and independent path for an active macro-zone approach, through which a major reduction in the number of plastic loops was obtained.

The contact/detachment problem utilized the well-known Signorini equations, rewritten in discrete form and solved through the LCP iterative strategy inside the SGBEM multidomain approach.

In the paper, all the typical advantages based on the employment of SGBEM, that is to say, high performance, accuracy of results and great stability, are shown.

Another important characteristic of the method is distinguishing the structural system into substructures: macroelements generally having large dimensions, where purely elastic behavior is assumed, and bem-elements generally having very small dimensions, where plastic strains may occur. The regularity conditions imposed between macroelements and bem-elements do not involve numerical instability, because of the evaluation in closed form of all the coefficients of the equation system, which regulate the two phenomena of elastoplasticity and contact/detachment.

\section{APPENDIX A}

In this Appendix the main novelties which make the SGBEM formulation highly competitive will be shown, including those treated in this paper.

\section{A.1. For all structural analyses}

The novelty of the SGBEM formulation lies in having formulated in [26], three typologies of structural analysis: displacement method, force method and mixed-value method, where the unknowns are respectively the displacements, the forces, and both the displacements and forces of the interface nodes between contiguous elements.

\section{A.2. For all structural analyses}

The novelty of the formulation introduced in [25] is the following: when the displacement method is employed and a subdivision into substructures is made, the novelty consists for each substructure, called a bem-elements, in writing an equation connecting the weighted tractions, evaluated along the boundary sides and associated with the nodes as a function of (a) the nodal displacement values of the same boundary through the introduction of a stiffness matrix and (b) the weighted tractions caused by boundary and body loads. This relation has the same structure as the nodal forces-nodal displacements relation, written within the finite element theory, but having the advantage that there is no constraint on the finite element type to be employed, because within the SGBEM approach the geometry and dimension of the bem-elements are quite generic.

When the mixed-value approach is utilized, as it is in the present paper, a pseudo-stiffness matrix, which relates the displacements and the weighted forces evaluated on the boundary sides with the nodal displacements and forces of the same boundary, is employed.

These relations are always utilized in all papers, which use these approaches within the SGBEM.

\section{A.3. For topics regarding the elastoplasticity}

The novelty in the elastoplastic analysis introduced in [8] is that in the present paper and in other papers on the same topic, a substructuring is employed through which the structure is subdivided into elements, called bem-elements, having the following:

- large dimensions with purely elastic behavior, and

- small dimensions in the zones where the rise of plastic strains is expected. 
Each element having large and small dimensions can have different physical and mechanical characteristics from the others.

This choice has a double advantage in comparison with the FEM because the number of variables comes down significantly. Indeed, we observe the following:

- The domain is discretized into elements having small dimensions only in the zones where one supposes that the plastic strains can arise, and elements having large dimensions where one supposes that the response is purely elastic.

- The geometry of each element, obtained by discretization, is quite generic.

It could be objected that the assemblage between bem-elements having large dimensions and bemelements having small dimensions involves numerical instability in the analysis. This does not happen because all the coefficients of the equation system are obtained in closed form [29]. This characteristic is a special quality of the SGBEM in comparison with other BEM methodologies. Further, the elements having small dimensions are assembled in restricted areas, called macro-zones. The plastic phenomenon occurs with a step-by-step process, where within each step the storage of plastic strains, regarding the bem-elements located in the potentially active macro-zones, takes place simultaneously in all the bem-elements where the yield domain is violated. At every step, the updating of the plastic strains and the consequent check of the stress state through the auto-stress matrix Z, also computed in closed form, are undertaken. The model adopted is a path-independent one which, because of the strategy utilized, has very low computational cost.

\section{A.4. For topics regarding contact detachment}

The novelty in the contact analysis introduced in [15] is that in the paper, the Signorini methodology is employed for contact detachment between two bodies having elastic behavior, where the simple check is made by considering appropriate conditions using the following quantities:

- generalized tractions associated with the boundary elements in contact, which can come off; and

- generalized displacements between separated boundary elements, which can come into contact.

\section{A.5. For the computational aspects}

The novelty regarding the computational aspects of the method [32] is the implementation of the Karnak.sGbem program (about $80 \mathrm{MB}$ with a strong graphic interface), which adopts the symmetric boundary element methodology, unique in its kind to our knowledge. In order to drastically reduce the CPU time, all the coefficients evaluated for each bem-elements, whether regarding the boundary quantities (boundary forces and displacements imposed in the constraint), or those the of domain (body forces and inelastic strains), are computed in closed form.

\section{A.6. For topics regarding body quantities}

The computational novelty in the analysis regarding the domain quantities in $[12,13]$ is that the presence of the domain integrals should greatly reduce the performance of the method and should render fruitless the positive aspects that the method can suggest. Therefore, to find in closed form, the coefficients regarding the body domain too (body forces and inelastic strains), preliminarily the transfer of these quantities onto the boundary is made through the radial integral method technique, introduced by Gao [10,11], and subsequently the boundary integrals are utilized.

Inside the this strategy, a difficulty arises in computing the coefficients regarding the inelastic strains because of the presence of the Bui free term, characterized by a domain integral having a singular kernel. One proceeds through a limit operation on the boundary, thus obtaining the weighted traction on each boundary element by means of two matrices whose coefficients are computed in closed form, one of the two matrices regarding the Bui free term evaluated on the boundary, the second one the slope of the boundary element being examined.

Overcoming this difficulty by using simple matrices makes the method highly effective. 


\section{A.7. The novelty of this paper}

Some advantages of the SGBEM method have been shown and applied in this paper, as for example:

- the use of a program where all the coefficients, whether those coming from the domain or those caused by boundary quantities, are computed in closed form;

- the use of a limited number of variables because it is possible to have elements having large dimensions with elastic behavior (checked by few boundary quantities) and macro-zones in a limited number where the elements have small dimensions and elastoplastic behavior; and

- the simplicity through which the contact-detachment process occurs between the boundaries of two contiguous elastoplastic bodies, involving only the updating of the dimension of each characteristic elastic matrix of the contiguous bodies by suppressing or adding only rows or columns.

\section{REFERENCES}

1. Telles JCF. The Boundary Element Method applied to Inelastic Problems. Springer: Berlin, 1983.

2. Beer G. An efficient numerical method for modelling initiation and propagation of cracks along material interfaces. International Journal for Numererical Methods in Engineering; 36(21):3579-3594.

3. Martin D, Aliabadi MH. Boundary element analysis of two-dimensional elastoplastic contact problems. Engineering Analysis with Boundary Elements 1998; 21:349-360.

4. Aliabadi MH, Martin D. Boundary element hyper-singular formulation for elastoplastic contact problem. International Journal for Numererical Methods in Engineering 2000; 48:995-1014.

5. Polizzotto C. Shakedown of elastic-plastic solids with frictionless unilateral contact boundary conditions. International Journal Mechanics Sciences 1997; 39:919-928.

6. Karami G. Boundary element analysis of elasto-plastic contact problems. Compututer and Structures 1991; 41:927-935.

7. Gun H. Boundary element analysis of 3-D elasto-plastic contact problems with friction. Computers and Structures 2004; 82:555-566.

8. Zito L, Cucco F, Parlavecchio E, Panzeca T. Incremental elastoplastic analysis for active macro-zones. International Journal for Numererical Methods in Engineering 2012; 91:1365-1385.

9. Panzeca T, Parlavecchio E, Zito L, Gao XW, Guo X. Lower bound limit analysis by BEM: convex optimization problem and incremental approach. Engineering Analysis with Boundary Elements 2013; 37:558-568.

10. Gao XW, Davies TG. An effective boundary element algorithm for 2D and 3D elastoplastic problem. International Journal of Solids and Structures 2000; 37:4987-5008.

11. Gao XW, Davies T G. Boundary element programming in mechanics. Cambridge University Press: Cambridge, UK, 2002.

12. Panzeca T, Terravecchia S, Zito L. Computational aspects in 2D SBEM analysis with domain inelastic actions. International Journal for Numerical Methods in Engineering 2010; 82:184-204.

13. Zito L, Parlavecchio E, Panzeca T. On the computational aspects of a symmetric multidomain BEM approach for elastoplastic analysis. Journal of Strain Analysis for Engineering Design 2011; 46:103-120.

14. Signorini A. Sopra alcune questioni di elastostatica. Atti della Società Italiana per il Progresso della Scienza 1993; 2:231-251.

15. Panzeca T, Salerno M, Terravecchia S, Zito L. The symmetric Boundary Element Method for unilateral contact problems. Computer Methods in Applied Mechanics and Engineering 2008; 197:2667-2679.

16. Zhang S, Zhu J. The boundary element-linear complementarity method for the Signorini problem. Engineering Analysis with Boundary Elements 2012; 36:112-117.

17. Salerno M, Terravecchia S, Zito L. Frictionless contact-detachment analysis: Iterative linear complementarity and quadratic programming approaches. Computational Mechanics 2013; 51:553-566.

18. Polizzotto C. An energy approach to the boundary element method. Part I: Elastic solids. Computer Methods in Applied Mechanics and Engineering 1988; 69:167-184.

19. Polizzotto C. An energy approach to the boundary element method. Part II: Elastic-plastic solids. Computer Methods in Applied Mechanics and Engineering 1988; 69:263-276.

20. Panzeca T, Salerno M. Macro-elements in the mixed boundary value problems. Computational Mechanics 2000; 26:437-446.

21. Terravecchia S. Revisited mixed-value method via symmetric BEM in the substructuring approach. Engineering Analysis with Boundary Elements 2012; 36:1865-1882.

22. Simo J, Hughes TJR. Computational Inelasticity. Springer-Verlag: New York, US, 1998.

23. Ortiz M, Martin JB. Symmetry-preserving return mapping algorthms and incrementally extremal paths: a unification of concepts. International Journal for Numerical Methods in Engineering 1989; 28:1839-1853.

24. Bilotta A, Casciaro R. A high-performance element for the analysis of 2D elastoplastic continua. Computer Methods in Applied Mechanics and Engineering 2007; 196:818-828. 
25. Panzeca T, Cucco F, Terravecchia S. Symmetric Boundary Element Method versus Finite Element Method. Computer Methods in Applied Mechanics and Engineering 2002; 191:3347-3367.

26. Panzeca T, Salerno M, Terravecchia S. Domain decomposition in the symmetric boundary element method analysis. Computational Mechanics 2002; 28:191-201.

27. Bonnet M. Regularized direct and indirect symmetric varational BIE formulation for three-dimensional elasticity. Engineering Analysis with Boundary Elements 2005; 15:93-102.

28. Frangi A, Novati G. Symmetric BE method in two-dimensional elasticity: evaluation of double integrals for curved elements. Computational Mechanics 1996; 19:58-68.

29. Terravecchia S. Closed form coefficients in the symmetric boundary element approach. Engineering Analysis with Boundary Elements 2006; 30:479-488.

30. Holzer S. How to deal with hypersingular integrals in the symmetric BEM. Communication Numerical Methods in Engineering 1993; 9:219-232.

31. Bui HD. Some remarks about the formulation of three-dimensional thermoelastoplastic problems by integral equation. International Journal of Solids and Structures 1978; 14:935-939.

32. Cucco F, Panzeca T, Terravecchia S. The program Karnak.sGbem Release 2.1: Palermo University, 2002.

33. Perez-Gavilan JJ, Aliabadi MH. A symmetric Galerkin BEM for Multi-connected bodies: A new apprach. Engineering Analysis with Boundary Elements 2001; 25:633-638.

34. Freddi F, Royer-Carfagni G. Symmetric Galerkin BEM for bodies with unconstrained contours. Computer Methods in Applied Mechanics and Engineering 2006; 195:961-981.

35. Vodicka R, Mantic V, Paris F. On the Removal of the non-uniqueness in the solution of the elastostatic problems by symmetric Galerkin BEM. International Journal of Solids and Structures 2006; 66:1884-1912. 